\title{
On a semilinear mixed fractional heat equation driven by fractional Brownian sheet
}

\author{
Dengfeng $X_{i a} a^{1,2}$ and Litan $\operatorname{Yan}^{1,3^{*}}$
}

\author{
*Correspondence: \\ litan-yan@hotmail.com \\ ${ }^{1}$ College of Information Science and \\ Technology, Donghua University, \\ Shanghai, 201620, P.R. China \\ ${ }^{3}$ Department of Mathematics, \\ College of Science, Donghua \\ University, Shanghai, 201620, \\ P.R. China \\ Full list of author information is \\ available at the end of the article
}

\begin{abstract}
In this paper, we consider the stochastic heat equation of the form

$$
\frac{\partial u}{\partial t}=\left(\Delta+\Delta_{\alpha}\right) u+\frac{\partial f}{\partial x}(t, x, u)+\frac{\partial^{2} W}{\partial t \partial x}
$$

where $W$ is a fractional Brownian sheet, $\Delta+\Delta_{\alpha}$ is a pseudo differential operator on $\mathbb{R}$ which gives rise to a Lévy process consisting of the sum of a Brownian motion and an independent symmetric $\alpha$-stable process, and $f:[0, T] \times \mathbb{R} \times \mathbb{R} \rightarrow \mathbb{R}$ is a nonlinear measurable function. We introduce the existence, uniqueness, Hölder regularity and density estimate of the solution.
\end{abstract}

MSC: $60 \mathrm{G} 22 ; 60 \mathrm{H07} ; 60 \mathrm{H} 15$

Keywords: stochastic partial differential equations; fractional Brownian sheet; Hölder regularity; density of the law; Malliavin calculus; $p$-variation

\section{Introduction}

Stochastic heat equations and fractional heat equations driven by fractional Brownian motion (sheet) are a recent research direction in probability theory and its applications. In Balan and Conus [1], Song [2], the authors considered intermittency for the fractional heat equation and a class of stochastic partial differential equations. In Chen et al. [3], Hu et al. [4], Hu, Lu and Nualart [5],the authors discussed the Feynman-Kac formula for fractional heat equations. In Bo et al. [6], Diop and Huang [7], Duncan et al. [8], Balan [9], Hu and Nualart [10], Liu and Yan [11], the authors introduced the stochastic heat equations with fractional white noises, and about the stochastic heat equations with fractional-colored noises we can see Jiang et al. [12, 13], Balan and Tudor [14, 15], Tudor [16] and the references therein. However, it is very limited to study the stochastic heat equations driven by the mixed fractional operator $\Delta+\Delta_{\alpha}$ and fractional Brownian sheet, where $\Delta_{\alpha}=-(-\Delta)^{\alpha / 2}$ is the fractional power of the Laplacian. On the other hand, many mathematical problems in physics and engineering with respect to systems and processes are represented by a kind of equations, more precisely fractional order differential equations driven by fractional noise. The increasing interest in this class of equations is motivated both by their applications to fluid dynamic traffic model, viscoelasticity, heat conduction in materials with memory, electrodynamics with memory and also because they can be employed to

(c) The Author(s) 2017. This article is distributed under the terms of the Creative Commons Attribution 4.0 International License (http://creativecommons.org/licenses/by/4.0/), which permits unrestricted use, distribution, and reproduction in any medium, provided you give appropriate credit to the original author(s) and the source, provide a link to the Creative Commons license, and indicate if changes were made. 
approach nonlinear conservation laws (see, for example, Sobczyk [17] and Droniou and Imbert [18]). Therefore, it seems interesting to handle the mixed fractional heat equations driven by fractional Brownian sheet. In this paper, we are concerned with the stochastic heat equation of the form

$$
\left\{\begin{array}{l}
\frac{\partial u}{\partial t}=\left(\Delta+\Delta_{\alpha}\right) u+\frac{\partial f}{\partial x}(t, x, u(t, x))+\frac{\partial^{2} W}{\partial t \partial x}, \quad t \in[0, T], x \in \mathbb{R} \\
u(0, x)=\vartheta(x), \quad x \in \mathbb{R}
\end{array}\right.
$$

with $0<\alpha<2$, where $W(t, x)$ is the fractional Brownian sheet and the nonlinear measurable function $f:[0, T] \times \mathbb{R} \times \mathbb{R} \rightarrow \mathbb{R}$ and the initial-value $\vartheta(x)$ satisfy the following assumptions:

Assumption 1 For some $p \geq 2$, we have

$$
\sup _{x \in \mathbb{R}} \mathbb{E}\left(|\vartheta(x)|^{p}\right)<+\infty
$$

and there is a constant $\theta \in(0,1)$ with $p \theta<1$ such that

$$
\sup _{x \in \mathbb{R}} \mathbb{E}\left(\left|\vartheta\left(x+x^{\prime}\right)-\vartheta(x)\right|^{p}\right)<C_{p}\left|x^{\prime}\right|^{p \theta}
$$

Assumption 2 For each $T>0$, there exists a constant $C>0$ such that

$$
\begin{aligned}
& |f(t, x, y)| \leq C(1+|y|), \\
& \left|f(t, x, y)-f\left(s, x^{\prime}, y^{\prime}\right)\right| \leq C\left(|t-s|+\left|x-x^{\prime}\right|+\left|y-y^{\prime}\right|\right)
\end{aligned}
$$

for all $(t, x, y) \in[0, T] \times \mathbb{R} \times \mathbb{R}$ and $x^{\prime}, y^{\prime} \in \mathbb{R}$.

The paper is organized as follows. Section 2 contains some preliminaries on the pseudo differential operator $\Delta+\Delta_{\alpha}$, the double-parameter fractional noises and the related Malliavin calculus. In Section 3, we study the existence and uniqueness of the mild solution to (1.1) by using a Picard approximation. In Section 4 we show the Hölder regularity of the solution $u(t, x)$. Section 5 is devoted to showing the existence of the density of $u(t, x)$ and we show that the law of $u(t, x)$ is absolutely continuous with respect to the Lebesgue measure on $\mathbb{R}$ by using Malliavin calculus.

\section{Preliminaries}

In this section, we briefly recall some basic results for Green function of the pseudo differential operator $\Delta+\Delta_{\alpha}$ and Malliavin calculus associated with fractional Brownian sheet. We refer to Chen et al. [19-23] and Nualart [24] and the references therein for more details. For convenience, in this paper we assume that $C$ is a positive constant depending only on $T, H, \alpha$ and its value may be different in different positions.

\subsection{On the pseudo differential operator $\Delta+\Delta_{\alpha}$}

It is well known that, for a second order elliptic differential operator $\mathscr{D}$ on $\mathbb{R}^{d}$ satisfying some natural conditions, there is a diffusion process $X$ on $\mathbb{R}^{d}$ such that $\mathscr{D}$ is its infinitesimal 
generator, and its transition density function is the fundamental solution of the equation

$$
\frac{\partial u}{\partial t}=\mathscr{D} u
$$

We also call the fundamental solution the heat kernel (Green function) of $\mathscr{D}$. For a large class of Markov processes with discontinuous sample paths, we also have such a correspondence, and such Markov processes have been widely used in various problems. In this one, an important Markov processes with discontinuous sample paths is (rotationally) symmetric $\alpha$-stable (Lévy) process with $0<\alpha \leq 2$.

A symmetric $\alpha$-stable process $X=\left\{X_{t}, t \geq 0, \mathbb{P}_{x}, x \in \mathbb{R}\right\}$ on $\mathbb{R}^{d}$ is a Lévy process such that

$$
\mathbb{E}_{x}\left[\exp \left\{i \zeta \cdot\left(X_{t}-X_{0}\right)\right\}\right]=\exp \left\{-t|\zeta|^{\alpha}\right\}
$$

for every $x, \zeta \in \mathbb{R}^{d}$, where $\mathbb{E}_{x}$ denotes the expectation with respect to $\mathbb{P}_{x}$. When $\alpha=2$, $X$ is a Brownian motion on $\mathbb{R}^{d}$ whose infinitesimal generator is the Laplacian $\Delta$. When $0<\alpha<2$, the infinitesimal generator of a symmetric $\alpha$-stable process $X$ is the fractional Laplacian $\Delta_{\alpha}=-(-\Delta)^{\alpha / 2}$, which is a nonlocal operator and it can be defined by

$$
\Delta_{\alpha} h(x)=\lim _{\delta \downarrow 0} \int_{\left\{x^{\prime} \in \mathbb{R}^{d}:\left|x^{\prime}-x\right|>\delta\right\}}\left(h\left(x^{\prime}\right)-h(x)\right) \frac{\mathcal{L}(d, \alpha)}{\left|x-x^{\prime}\right|^{d+\alpha}} d x^{\prime}
$$

where $\mathcal{L}(d, \alpha):=\alpha 2^{\alpha-1} \pi^{-\frac{d}{2}} \Gamma\left(\frac{d+\alpha}{2}\right) / \Gamma\left(1-\frac{\alpha}{2}\right)$ and $\Gamma$ denotes the classical Gamma function. In this paper, we consider only the case $d=1$.

Let now $X^{\alpha}$ be a real value $\alpha$-stable process with $0<\alpha<2$ and let $B$ be a real value Brownian motion independent of $X^{\alpha}$. Define the process $X$ by

$$
X_{t}:=B_{t}+X_{t}^{\alpha}, \quad t \geq 0
$$

Then the infinitesimal generator of $X$ is $\Delta+\Delta_{\alpha}$ and

$$
\mathbb{E}_{x}\left[\exp \left\{i \zeta\left(X_{t}-X_{0}\right)\right\}\right]=\exp \left\{-t\left(|\zeta|^{2}+|\zeta|^{\alpha}\right)\right\}
$$

for every $x, \zeta \in \mathbb{R}^{d}$. Denote by $G_{\alpha}(t, x)$ the fundamental solution of the equation

$$
\frac{\partial u}{\partial t}=\left(\Delta+\Delta_{\alpha}\right) u
$$

(or equivalently the heat kernel of $\Delta+\Delta_{\alpha}$ ). It follows from Chen et al. [23] that

$$
\begin{aligned}
& C_{1}^{-1}\left((t-s)^{-\frac{1}{2}} e^{-\frac{C_{2}|x-y|^{2}}{(t-s)}}+(t-s)^{-\frac{1}{2}} \wedge \frac{t-s}{|x-y|^{1+\alpha}}\right) \\
& \quad \leq G_{\alpha}(s, y ; t, x) \leq C_{1}\left((t-s)^{-\frac{1}{2}} e^{-\frac{|x-y|^{2}}{C_{2}(t-s)}}+(t-s)^{-\frac{1}{2}} \wedge \frac{t-s}{|x-y|^{1+\alpha}}\right)
\end{aligned}
$$

for all $t>s>0, x, y \in \mathbb{R}^{d}$ and some constants $C_{1}, C_{2}>1$, where $a_{1} \wedge a_{2}:=\min \left\{a_{1}, a_{2}\right\}$ for $a_{1}, a_{2} \in \mathbb{R}$ and $G_{\alpha}(s, y ; t, x):=G_{\alpha}(t-s, x-y)$. 


\subsection{Malliavin calculus}

Recall that a fractional Brownian sheet defined on a probability space $(\Omega, \mathscr{F}, P)$ with indices $H_{1}, H_{2} \in(0,1), W=\{W(t, x), t \in[0, T], x \in \mathbb{R}\}$ is a Gaussian random field with $W(0,0)=0$ and

$$
E W(t, x)=0, \quad E(W(t, x) W(s, y))=R_{H_{1}}(s, t) R_{H_{2}}(x, y)
$$

for all $s, t>0, x, y \in \mathbb{R}$, where

$$
R_{H_{j}}(u, v)=\frac{1}{2}\left(|u|^{2 H_{j}}+|v|^{2 H_{j}}-|u-v|^{2 H_{j}}\right), \quad j=1,2 ; u, v \in \mathbb{R} .
$$

Let $\mathcal{H}$ be the completion of the linear space $\mathcal{I}$ generated by the indicator functions $1_{(s, t] \times(x, y]}$ on $[0, T] \times \mathbb{R}$ with respect to the scalar product

$$
\left\langle 1_{[0, t] \times[0, x]}, 1_{[0, s] \times[0, y]}\right\rangle_{\mathcal{H}}=R_{H_{1}}(s, t) R_{H_{2}}(x, y) .
$$

The following embedding property follows from Bo et al. [6] (see also Jiang et al. [13] and Wei [25]).

Proposition 2.1 For $H>\frac{1}{2}$ we have

$$
L^{\frac{1}{H}}([0, T] \times \mathbb{R}) \subset \mathcal{H} .
$$

Define the mapping $W(g)$ between $\mathcal{I}$ and the Gaussian space associated with $W$ by

$$
g=1_{[0, t] \times[0, x]} \longmapsto \int_{0}^{T} \int_{\mathbb{R}} g(s, x) W(d s, d y)=W(t, x) .
$$

Then it is an isometry and it can be extended to $\mathcal{H}$, which is called the Wiener integral of $g$ with respect to $W$. Denote

$$
\Xi_{H}(t, s ; x, y)=4 H_{1} H_{2}\left(2 H_{1}-1\right)\left(2 H_{2}-1\right)|t-s|^{2 H_{1}-2}|x-y|^{2 H_{2}-2}
$$

for any $0 \leq s<t \leq T$ and $x, y \in \mathbb{R}$.

Proposition 2.2 For $\varphi, \psi \in \mathcal{H}$, we have $E[W(\mu)]=0$ and

$$
E[W(\varphi) W(\psi)]=\int_{[0, T]^{2}} d s d t \int_{\mathbb{R}^{2}} \varphi(s, x) \psi(t, y) \Xi_{H}(s, t ; x, y) d y d x
$$

Proposition 2.3 If $H \in\left(\frac{1}{2}, 1\right)$ and $\varphi, \psi \in L^{\frac{1}{H}}([a, b])$, then

$$
\int_{[a, b]^{2}} \varphi(x) \psi(y)|x-y|^{2 H-2} d x d y \leq C\|\varphi\|_{L^{\frac{1}{H}([a, b])}}\|\psi\|_{L^{\frac{1}{H}}([a, b])} .
$$

Consider now the set $\mathcal{C}$ of smooth and cylindrical functional

$$
\digamma=f\left(W\left(g_{1}\right), \ldots, W\left(g_{n}\right)\right)
$$


where the function $f$ and its derivatives of all orders are bounded and $g_{i} \in \mathcal{H}, i=1, \ldots, n$. Define the derivative operator $D F$ (the Malliavin derivative) by

$$
D \digamma:=\sum_{j=1}^{n} \frac{\partial f}{\partial x_{j}} W\left(g_{1}\right), \ldots, W\left(g_{n}\right) g_{j}
$$

for the functional $\digamma$ of the form (2.2). Then $D$ is a close operator from $L^{2}(\Omega)$ into $L^{2}(\Omega ; \mathcal{H})$. Denote $D_{h} \digamma=\langle D \digamma, h\rangle_{\mathcal{H}}$. Let $\mathbb{D}_{h}$ and $\mathbb{D}^{1,2}$ be the closures of $\mathcal{C}$ with respect to the norms

$$
\|\digamma\|_{h}=\sqrt{E\left[|\digamma|^{2}+\left\|D_{h} \digamma\right\|^{2}\right]}
$$

for $h \in \mathcal{H}$ and

$$
\|\digamma\|_{1,2}=\left(E|\digamma|^{2}+E\|D \digamma\|_{\mathcal{H}}^{2}\right)^{1 / 2}
$$

respectively. Then $\mathbb{D}^{1,2}$ is the domain of $D$ on $L^{2}(\Omega)$ and

$$
\digamma \in \mathbb{D}^{1,2} \Longleftrightarrow \digamma \in \mathbb{D}_{h_{n}}
$$

for each $n \in \mathbb{N}$, if $\sum_{n=1}^{\infty} E\left|D_{h_{n}} \digamma\right|^{2}<+\infty$, where $\left\{h_{n}, n \geq 1\right\}$ is an orthogonal basis of $\mathcal{H}$.

The divergence operator (integral) $\delta$ is defined as the adjoint of $D$. A random variable $u \in L^{2}(\Omega ; \mathcal{H})$ belongs to the domain $\operatorname{Dom}(\delta)$ of $\delta$, provided

$$
E\left|\langle D \digamma, u\rangle_{\mathcal{H}}\right| \leq C\|\digamma\|_{L^{2}(\Omega)}
$$

for all $\digamma \in \mathcal{C}$. Thus, $\delta(u)$ can be determined by the next duality relationship:

$$
E\langle D \digamma, u\rangle_{\mathcal{H}}=E(\digamma \delta(u)), \quad u \in \mathbb{D}^{1,2} .
$$

We will also use the next notations:

$$
\delta(u)=\int_{0}^{T} \int_{\mathbb{R}} u(s, x) W(d s, d x)
$$

and

$$
\delta\left(u 1_{[0, t] \times A}\right)=\int_{0}^{t} \int_{A} u(s, x) W(d s, d x) .
$$

By using Malliavin calculus for stochastic partial differential equations (abbr. SPDEs) driven by fractional noises, we can get the following propositions (see, e.g., Wei [25] and Jiang et al. [13]).

Proposition 2.4 Let $\mathscr{F}_{N}:=\sigma\{W(M), M \subset N\}$ for $N \in \mathscr{B}([0, T] \times \mathbb{R})$, and let the random variable $Y$ be square integrable. If $Y$ is measurable with respect the $\sigma$-field $\mathscr{F}_{N^{c}}$, then

$$
D Y 1_{N}=0,
$$

almost surely. 
Proposition 2.5 For a random variable $Y$ belonging to $\mathbb{D}^{1,2}$, if $\|D Y\|_{\mathcal{H}}^{2}>0$ almost surely, the law of $Y$ is absolutely continuous with respect to the Lebesgue measure.

\section{Existence and uniqueness of the solution}

Given a filtered probability space $\left(\Omega, \mathscr{F},\left(\mathscr{F}_{t}\right)_{t \geq 0}, P\right)$ with the natural filtration $\left(\mathscr{F}_{t}\right)_{t \geq 0}$ of $W$. In this section, the Cauchy problem (1.1) will be discussed. By using the heat kernel $G_{\alpha}(s, y ; t, x)$ of $\Delta+\Delta_{\alpha}$, as usual (see, e.g., Walsh [26]) we say that the stochastic field

$$
u:[0, T] \times \mathbb{R} \times \Omega \longmapsto \mathbb{R}
$$

is a mild solution to (1.1) if

$$
\begin{aligned}
u(t, x)= & \int_{\mathbb{R}} G_{\alpha}(0, y ; t, x) \vartheta(y) d y+\int_{0}^{t} \int_{\mathbb{R}} G_{\alpha}(s, y ; t, x) W(d s, d y) \\
& +\int_{0}^{t} \int_{\mathbb{R}} \frac{\partial G_{\alpha}}{\partial y}(s, y ; t, x) f(s, y, u(s, y)) d y d s
\end{aligned}
$$

for all $t \geq 0$ and $x \in \mathbb{R}$. Now we can state the main result in this section, and its proof could be derived by using some estimates of the heat kernel $G_{\alpha}(s, y ; t, x)$ and some properties of the stochastic integral

$$
\int_{0}^{t} \int_{\mathbb{R}} G_{\alpha}(s, y ; t, x) W(d s, d y) .
$$

Theorem 3.1 Under Assumptions 1 and 2, equation (3.1) admits a unique solution $u=$ $\{u(t, x),(t, x) \in[0, T] \times \mathbb{R}\}$ such that

$$
\sup _{t \in[0, T], x \in \mathbb{R}} E|u(t, x)|^{p}<+\infty
$$

for all $\alpha \in(0,2)$ and $p \geq 2$.

Proof We first use Picard's approximation to get a solution to (3.1) and then we show that the solution is unique. This proof will be decomposed into three steps, and we define

$$
\left\{\begin{aligned}
u_{0}(t, x)=\int_{\mathbb{R}} & G_{\alpha}(0, y ; t, x) \vartheta(y) d y \\
u_{n+1}(t, x)= & u_{0}(t, x)+\int_{0}^{t} \int_{\mathbb{R}} G_{\alpha}(s, y ; t, x) W(d y, d s) \\
& +\int_{0}^{t} \int_{\mathbb{R}} \frac{\partial G_{\alpha}}{\partial y}(s, y ; t, x) f\left(s, y, u_{n}(s, y)\right) d y d s
\end{aligned}\right.
$$

for all $t \geq 0, x \in \mathbb{R}$ and $n \in \mathbb{N}=\{0,1,2, \ldots\}$.

Step I. We prove that

$$
\sup _{n \in \mathbb{N}} \sup _{t \in[0, T], x \in \mathbb{R}} E\left|u_{n}(t, x)\right|^{p}<+\infty .
$$

By Hölder's inequality and Assumption 1, we get

$$
\begin{aligned}
E\left|u_{0}(t, x)\right|^{p} & \leq E\left(\left(\int_{\mathbb{R}}\left|G_{\alpha}(0, y ; t, x)\right| d y\right)^{p-1} \int_{\mathbb{R}}\left|G_{\alpha}(0, y ; t, x)\right||\vartheta(y)|^{p} d y\right) \\
& \leq \sup _{x \in \mathbb{R}} E|\vartheta(x)|^{p}\left(\int_{\mathbb{R}}\left|G_{\alpha}(0, y ; t, x)\right| d y\right)^{p}
\end{aligned}
$$


for all $p \geq 2$. Notice that (2.1) implies that

$$
\sup _{t \in[0, T], x \in \mathbb{R}} \int_{\mathbb{R}}\left|G_{\alpha}(0, y ; t, x)\right| d y<+\infty
$$

We see that $\sup _{t \in[0, T], x \in \mathbb{R}} E\left|u_{0}(t, x)\right|^{p}<+\infty$.

On the other hand, for each $n \geq 1$ and $p \geq 2$ we denote

$$
\begin{aligned}
& \Phi_{p, n}(t, x)=E\left|\int_{0}^{t} \int_{\mathbb{R}} G_{\alpha}(s, y ; t, x) W(d y, d s)\right|^{p}, \\
& \Psi_{p, n}(t, x)=E\left|\int_{0}^{t} \int_{\mathbb{R}} \frac{\partial G_{\alpha}}{\partial y}(s, y ; t, x) f\left(s, y, u_{n}(s, y)\right) d y d s\right|^{p} .
\end{aligned}
$$

By (3.2) it follows that

$$
E\left|u_{n+1}(t, x)\right|^{p} \leq C\left(E\left|u_{0}(t, x)\right|^{p}+\Phi_{p, n}(t, x)+\Psi_{p, n}(t, x)\right) .
$$

We need to estimate $\Phi_{p, n}(t, x)$ and $\Psi_{p, n}(t, x)$. Clearly, we have

$$
\int_{\mathbb{R}}(t-s)^{-\frac{1}{2 H_{2}}} e^{-\frac{C|x-y|^{2}}{t-s}} d y \leq C(t-s)^{-\frac{1}{2 H_{2}}+\frac{1}{2}}
$$

and

$$
\int_{\mathbb{R}}\left((t-s)^{-\frac{1}{2}} \wedge \frac{(t-s)}{|x-y|^{1+\alpha}}\right)^{\frac{1}{H_{2}}} d y \leq C(t-s)^{-\frac{1}{2 H_{2}}+\frac{3}{2(1+\alpha)}}
$$

for all $t>s>0$. It follows that

$$
\begin{aligned}
& \left\|G_{\alpha}(s, \cdot ; t, x)\right\|_{L^{\frac{1}{H_{2}}}(\mathbb{R})} \\
& \quad=\left(\int_{\mathbb{R}} G_{\alpha}(s, y ; t, x)^{\frac{1}{H_{2}}} d y\right)^{H_{2}} \\
& \quad \leq C\left(\int_{\mathbb{R}}\left((t-s)^{-\frac{1}{2}} e^{-\frac{|x-y|^{2}}{C_{2}(t-s)}}+(t-s)^{-\frac{1}{2}} \wedge \frac{(t-s)}{|x-y|^{1+\alpha}}\right)^{\frac{1}{H_{2}}} d y\right)^{H_{2}} \\
& \quad \leq C\left(\int_{\mathbb{R}}\left((t-s)^{-\frac{1}{2}} e^{-\frac{|x-y|^{2}}{C_{2}(t-s)}}\right)^{\frac{1}{H_{2}}} d y+\int_{\mathbb{R}}\left((t-s)^{-\frac{1}{2}} \wedge \frac{(t-s)}{|x-y|^{1+\alpha}}\right)^{\frac{1}{H_{2}}} d y\right)^{H_{2}} \\
& \quad \leq C\left((t-s)^{-\frac{1}{2}+\frac{H_{2}}{2}}+(t-s)^{-\frac{1}{2}+\frac{3 H_{2}}{2(1+\alpha)}}\right) \leq C(t-s)^{-\frac{1}{2}+\frac{H_{2}}{2}},
\end{aligned}
$$

which implies that

$$
\begin{aligned}
& \Phi_{p, n}(t, x) \\
& \quad=E\left|\int_{0}^{t} \int_{\mathbb{R}} G_{\alpha}(r, z ; t, x) W(d r, d z)\right|^{p} \\
& \quad \leq C\left(\int_{0}^{t} \int_{0}^{t} d r_{1} d r_{2} \int_{\mathbb{R}^{2}} G_{\alpha}\left(r_{1}, z_{1} ; t, x\right) \Xi\left(r_{1}, r_{2} ; z_{1}, z_{2}\right) G_{\alpha}\left(r_{2}, z_{2} ; t, x\right) d z_{1} d z_{2}\right)^{\frac{p}{2}}
\end{aligned}
$$




$$
\begin{aligned}
= & C\left(\int_{0}^{t} \int_{0}^{t}\left|r_{1}-r_{2}\right|^{2 H_{1}-2} d r_{1} d r_{2} \int_{\mathbb{R}^{2}}\left|z_{1}-z_{2}\right|^{2 H_{2}-2}\right. \\
& \left.\cdot G_{\alpha}\left(r_{1}, z_{1} ; t, x\right) G_{\alpha}\left(r_{2}, z_{2} ; t, x\right) d z_{1} d z_{2}\right)^{\frac{p}{2}} \\
\leq & C\left(\int_{0}^{t} \int_{0}^{t}\left|r_{1}-r_{2}\right|^{2 H_{1}-2}\left\|G_{\alpha}\left(r_{1}, \cdot ; t, x\right)\right\|_{L^{\frac{1}{H_{2}}}(\mathbb{R})}\left\|G_{\alpha}\left(r_{2}, \cdot ; t, x\right)\right\|_{L^{\frac{1}{H_{2}}(\mathbb{R})}} d r_{1} d r_{2}\right)^{\frac{p}{2}} \\
\leq & C\left(\int_{0}^{T}\left(\left\|G_{\alpha}(r, \cdot ; t, x)\right\|_{L^{\frac{1}{H_{2}}(\mathbb{R})}}\right)^{\frac{1}{H_{1}}} d r\right)^{p H_{1}} \leq C<+\infty
\end{aligned}
$$

by Propositions 2.1, 2.2, and (2.1). Similarly, by the Hölder inequality we get

$$
\begin{aligned}
\Psi_{p, n}(t, x) \leq & C\left(\int_{0}^{t} \int_{\mathbb{R}}\left|\frac{\partial G_{\alpha}}{\partial y}(s, y ; t, x)\right| d y d s\right)^{p-1} \\
& \cdot \int_{0}^{t} \int_{\mathbb{R}}\left(E\left|f\left(s, y, u_{n}(s, y)\right)\right|^{p}\right)\left|\frac{\partial G_{\alpha}}{\partial y}(s, y ; t, x)\right| d y d s \\
\leq & C \int_{0}^{t} \int_{\mathbb{R}}\left|\frac{\partial G_{\alpha}}{\partial y}(s, y ; t, x)\right| d y d s \\
& +C \int_{0}^{t} \int_{\mathbb{R}} E\left|u_{n}(s, y)\right|^{p}\left|\frac{\partial G_{\alpha}}{\partial y}(s, y ; t, x)\right| d y d s \\
\leq & C \int_{0}^{t} \int_{\mathbb{R}}\left|\frac{\partial G_{\alpha}}{\partial y}(s, y ; t, x)\right| d y d s \\
& +C \int_{0}^{t} \sup _{y \in \mathbb{R}} E\left|u_{n}(s, y)\right|^{p} d s \int_{\mathbb{R}}\left|\frac{\partial G_{\alpha}}{\partial y}(s, y ; t, x)\right| d y .
\end{aligned}
$$

Denote $g_{x}(t-s)=\int_{\mathbb{R}}\left|\frac{\partial G_{\alpha}}{\partial y}(s, y ; t, x)\right| d y, \mathbb{D}_{x}=\left\{z \in \mathbb{R}|| x-z \mid<(t-s)^{\frac{3}{2(1+\alpha)}}\right\}$, and

$$
\begin{aligned}
& G_{1, \alpha}(s, y ; t, x):=(t-s)^{-\frac{1}{2}} e^{-\frac{|x-y|^{2}}{C_{2}(t-s)}}+(t-s)^{-\frac{1}{2}}, \\
& G_{2, \alpha}(s, y ; t, x):=(t-s)^{-\frac{1}{2}} e^{-\frac{|x-y|^{2}}{C_{2}(t-s)}}+\frac{t-s}{|x-y|^{1+\alpha}}
\end{aligned}
$$

for all $t>s>0$ and $x, y \in \mathbb{R}$. It follows from (3.6), (3.7), and (2.1) that

$$
\sup _{x \in \mathbb{R}} E\left|u_{n+1}(t, x)\right|^{p} \leq C+\int_{0}^{t} g_{x}(t-s) d s+\int_{0}^{t} \sup _{y \in \mathbb{R}} E\left|u_{n}(s, y)\right|^{p} g_{x}(t-s) d s
$$

and

$$
\begin{aligned}
\int_{\mathbb{R}}\left|\frac{\partial G_{\alpha}}{\partial y}(s, y ; t, x)\right| d y \leq & \int_{\mathbb{D}_{x}}\left|\frac{\partial G_{1, \alpha}}{\partial y}(s, y ; t, x)\right| d y+\int_{\mathbb{\mathbb { D }}_{x}}\left|\frac{\partial G_{2, \alpha}}{\partial y}(s, y ; t, x)\right| d y \\
= & \int_{\mathbb{D}_{x}}\left|(t-s)^{-\frac{1}{2}} e^{-\frac{|x-y|^{2}}{C_{2}(t-s)}}\left(-\frac{2|x-y|}{C_{2}(t-s)}\right)\right| d y \\
& +\int_{\overline{\mathbb{D}}_{x}}\left|(t-s)^{-\frac{1}{2}} e^{-\frac{|x-y|^{2}}{C_{2}(t-s)}}\left(-\frac{2|x-y|}{C_{2}(t-s)}\right)-(1+\alpha) \frac{t-s}{|x-y|^{2+\alpha}}\right| d y \\
\leq & C|t-s|^{-\frac{1}{2}} .
\end{aligned}
$$


Combining this with Lemma 15 in Dalang [27], we get

$$
\sup _{n \in \mathbb{N}} \sup _{t \in[0, T], x \in \mathbb{R}} E\left|u_{n}(t, x)\right|^{p}<+\infty
$$

Step II. We prove that $\left\{u_{n}(t, x)\right\}_{n \in \mathbb{N}}$ converges in $L^{p}(\Omega)$ for any $p \geq 2$. For $n \geq 2$, we have

$$
\begin{aligned}
E\left(\left|u_{n+1}(t, x)-u_{n}(t, x)\right|^{p}\right) & \\
= & E\left(\mid \int_{0}^{t} \int_{\mathbb{R}} \frac{\partial G_{\alpha}}{\partial y}(s, y ; t, x) f\left(s, y, u_{n}(s, y)\right) d y d s\right. \\
& \left.-\left.\int_{0}^{t} \int_{\mathbb{R}} \frac{\partial G_{\alpha}}{\partial y}(s, y ; t, x) f\left(s, y, u_{n-1}(s, y)\right) d y d s\right|^{p}\right) \\
\leq & C \int_{0}^{t}\left(\int_{\mathbb{R}}\left|\frac{\partial G_{\alpha}}{\partial y}(s, y ; t, x)\right| d y\right) E\left|u_{n}(s, y)-u_{n-1}(s, y)\right|^{p} d s \\
\leq & C \int_{0}^{t}\left(\int_{\mathbb{R}}\left|\frac{\partial G_{\alpha}}{\partial y}(s, y ; t, x)\right| d y\right) \sup _{y \in \mathbb{R}} E\left|u_{n}(s, y)-u_{n-1}(s, y)\right|^{p} d s
\end{aligned}
$$

and

$$
\sup _{y \in \mathbb{R}} E\left|u_{1}(s, y)-u_{0}(s, y)\right|^{p} \leq C_{p}\left(E\left|u_{0}(s, y)\right|^{p}+E\left|u_{1}(s, y)\right|^{p}\right)<+\infty .
$$

Combining this with Gronwall's inequality, we get

$$
\sum_{n \in \mathbb{N}} \sup _{t \in[0, T], x \in \mathbb{R}} E\left(\left|u_{n+1}(t, x)-u_{n}(t, x)\right|^{p}\right)<+\infty
$$

which implies that $\left\{u_{n}(t, x)\right\}_{n \geq 0}$ is a Cauchy sequence in $L^{p}(\Omega)$. Define

$$
u(t, x):=\lim _{n \rightarrow+\infty} u_{n}(t, x)
$$

in $L^{p}(\Omega)$. Then we have

$$
\sup _{t \in[0, T], x \in \mathbb{R}} E|u(t, x)|^{p}<+\infty
$$

for each $(t, x) \in[0, T] \times \mathbb{R}$. Taking $n \rightarrow+\infty$ in $L^{p}(\Omega)$ for (3.2), we see that $\{u(t, x):(t, x) \in$ $[0, t] \times \mathbb{R}\}$ satisfies $(3.1)$.

Step III. We prove the uniqueness of the solution. Let $u$ and $\hat{u}$ be the two mild solutions of (1.1), then

$$
\begin{aligned}
E\left(|u(t, x)-\hat{u}(t, x)|^{p}\right) & \\
= & E\left(\mid \int_{0}^{t} \int_{\mathbb{R}} \frac{\partial G_{\alpha}}{\partial y}(s, y ; t, x) f(s, y, u(s, y)) d y d s\right. \\
& \left.-\left.\int_{0}^{t} \int_{\mathbb{R}} \frac{\partial G_{\alpha}}{\partial y}(s, y ; t, x) f(s, y, \hat{u}(s, y)) d y d s\right|^{p}\right) \\
\leq & C \int_{0}^{t}\left(\int_{\mathbb{R}}\left|\frac{\partial G_{\alpha}}{\partial y}(s, y ; t, x)\right| d y\right) E|u(s, y)-\hat{u}(s, y)|^{p} d s \\
\leq & C \int_{0}^{t} \sup _{y \in \mathbb{R}} E|u(s, y)-\hat{u}(s, y)|^{p} \int_{\mathbb{R}}\left|\frac{\partial G_{\alpha}}{\partial y}(s, y ; t, x)\right| d y d s .
\end{aligned}
$$


It follows from Gronwall's inequality that

$$
\sup _{t \in[0, T], x \in \mathbb{R}} E|u(t, x)-\hat{u}(t, x)|^{p}=0
$$

for all $T>0$. Thus, we have completed the proof of the theorem.

\section{Hölder regularity and $p$-variation of the solution}

In this section we expound and prove the next theorem, which gives the Hölder regularity of the solution $u=\{u(t, x),(t, x) \in[0, T] \times \mathbb{R}\}$ to $(3.1)$.

Theorem 4.1 Let $H_{1}, H_{2} \in\left(\frac{1}{2}, 1\right)$ and $\alpha \in(1,2)$. Under Assumptions 1 and 2 , the solution $u(t, x)$ has a continuous version which is $\gamma$-Hölder continuous in $t$ with $\gamma \in\left(0, \vartheta_{1}\right)$ and $\nu$-Hölder continuous in $x$ with $v \in\left(0, \vartheta_{2}\right)$, where

$$
\vartheta_{1}:=\min \left\{\frac{\theta}{2}, \frac{2 H_{1}-1}{2}+\frac{H_{2}}{2}\right\}, \quad \vartheta_{2}:=\min \left\{\theta, H_{2}\right\} .
$$

In order to show that the theorem holds we need two lemmas.

Lemma 4.1 We have

$$
\int_{\mathbb{R}}\left(\left|\frac{\partial G_{\alpha}}{\partial t}(r, z ; t, x)\right|^{\theta_{1}}\left|G_{\alpha}(r, z ; t, x)\right|^{1-\theta_{1}}\right)^{\frac{1}{H_{2}}} d z \leq C(t-r)^{\frac{1}{2}-\frac{2 \theta_{1}+1}{2 H_{2}}}
$$

for all $0<r<t \leq T, x \in \mathbb{R}$ and $\theta_{1} \in(0,1)$. Moreover, when $0<\theta_{1}<\frac{2 H_{1}-1}{2}+\frac{H_{2}}{2}$, we have also

$$
\int_{0}^{t}\left(\int_{\mathbb{R}}\left(\left|\frac{\partial G_{\alpha}}{\partial t}(r, z ; t, x)\right|^{\theta_{1}}\left|G_{\alpha}(r, z ; t, x)\right|^{1-\theta_{1}}\right)^{\frac{1}{H_{2}}} d z\right)^{\frac{H_{2}}{H_{1}}} d r \leq C
$$

for all $t \in[0, T]$ and $x \in \mathbb{R}$.

Proof Given $t>r$ and $z \in \mathbb{R}$. Recall that $\mathbb{D}_{x}=\left\{y \in \mathbb{R}|| x-y \mid<(t-r)^{\frac{3}{2(1+\alpha)}}\right\}$,

$$
\begin{aligned}
\int_{\mathbb{R}}\left(\left|\frac{\partial G_{\alpha}}{\partial t}(r, z ; t, x)\right|^{\theta_{1}}\left|G_{\alpha}(r, z ; t, x)\right|^{1-\theta_{1}}\right)^{\frac{1}{H_{2}}} d z \\
\leq \int_{\mathbb{D}_{z}}\left|\frac{\partial G_{1, \alpha}}{\partial t}(r, z ; t, x)\right|^{\frac{\theta_{1}}{H_{2}}}\left|G_{1, \alpha}(r, z ; t, x)\right|^{\frac{1-\theta_{1}}{H_{2}}} d z \\
\quad+\int_{\overline{\mathbb{D}_{z}}}\left|\frac{\partial G_{2, \alpha}}{\partial t}(r, z ; t, x)\right|^{\frac{\theta_{1}}{H_{2}}}\left|G_{2, \alpha}(r, z ; t, x)\right|^{\frac{1-\theta_{1}}{H_{2}}} d z \\
\equiv A_{2,1,1,1}^{1}(t, r, x)+A_{2,1,1,2}^{1}(t, r, x)
\end{aligned}
$$

for all $0<r<t \leq T$ and $x \in \mathbb{R}$. Clearly, we have

$$
\begin{aligned}
& A_{2,1,1,1}^{1}(t, r, x) \\
& \quad=\int_{\mathbb{D}_{z}}\left|-\frac{1}{2}(t-r)^{-\frac{3}{2}} e^{-\frac{C|x-z|^{2}}{t-r}}+(t-r)^{-\frac{1}{2}} \frac{C|x-z|^{2}}{(t-r)^{2}} e^{-\frac{C|x-z|^{2}}{t-r}}-\frac{1}{2}(t-r)^{-\frac{3}{2}}\right|^{\frac{\theta_{1}}{H_{2}}}
\end{aligned}
$$




$$
\begin{aligned}
& \cdot\left|(t-r)^{-\frac{1}{2}} e^{-\frac{C|x-z|^{2}}{t-r}}+(t-r)^{-\frac{1}{2}}\right|^{\frac{1-\theta_{1}}{H_{2}}} d z \\
\leq & (t-r)^{-\frac{3 \theta_{1}}{2 H_{2}}-\frac{1-\theta_{1}}{2 H_{2}}} \int_{\mathbb{D}_{z}}\left|e^{-\frac{C|x-z|^{2}}{t-r}}+\frac{|x-z|^{2}}{t-r} e^{-\frac{C|x-z|^{2}}{t-r}}+1\right|^{\frac{\theta_{1}}{H_{2}}}\left|e^{-\frac{C|x-z|^{2}}{t-r}}+1\right|^{\frac{1-\theta_{1}}{H_{2}}} d z \\
\leq & C(t-r)^{-\frac{3 \theta_{1}}{2 H_{2}}-\frac{1-\theta_{1}}{2 H_{2}}} \int_{\mathbb{D}_{z}} d z \leq C(t-r)^{\frac{3}{2(1+\alpha)}-\frac{1+2 \theta_{1}}{2 H_{2}}}
\end{aligned}
$$

by the fact $x^{2} e^{-x^{2}} \leq 1$ and

$$
\begin{aligned}
A_{2,1,1,2}^{1}(t, r, x) & \\
= & \int_{\overline{\mathbb{D}}_{z}}\left|-\frac{1}{2}(t-r)^{-\frac{3}{2}} e^{-\frac{|x-z|^{2}}{C_{2}(t-r)}}+(t-r)^{-\frac{1}{2}} \frac{|x-z|^{2}}{C_{2}(t-r)} e^{-\frac{|x-z|^{2}}{C_{2}(t-r)}}+\frac{1}{|x-z|^{1+\alpha}}\right|^{\frac{\theta_{1}}{H_{2}}} \\
& \cdot\left|(t-r)^{-\frac{1}{2}} e^{-\frac{|x-z|^{2}}{C_{2}(t-r)}}+\frac{t-r}{|x-z|^{1+\alpha}}\right|^{\frac{1-\theta_{1}}{H_{2}}} d z \\
\leq & C(t-r)^{-\frac{3 \theta_{1}}{2 H_{2}}-\frac{1-\theta_{1}}{2 H_{2}}} \int_{\overline{\mathbb{D}}_{z}}\left|e^{-\frac{|x-z|^{2}}{C_{2}(t-r)}}+\right| x-\left.z\right|^{2} e^{-\frac{|x-z|^{2}}{C_{2}(t-r)}}+\left.\frac{(t-r)^{3 / 2}}{|x-z|^{1+\alpha}}\right|^{\frac{\theta_{1}}{H_{2}}} \\
& \cdot\left|e^{-\frac{|x-z|^{2}}{C_{2}(t-r)}}+\frac{(t-r)^{3 / 2}}{|x-z|^{1+\alpha}}\right|^{\frac{1-\theta_{1}}{H_{2}}} d z \\
\leq & C(t-r)^{-\frac{3 \theta_{1}}{2 H_{2}}-\frac{1-\theta_{1}}{2 H_{2}}} \int_{\overline{\mathbb{D}}_{z}}\left(e^{-\frac{C(x-z)^{2}}{t-r}}+|x-z|^{\frac{2 \theta_{1}}{H_{2}}} e^{-\frac{C(x-z)^{2}}{t-r}}+\frac{(t-r)^{\frac{3 \theta_{1}}{2 H_{2}}}}{|x-z|^{\frac{\theta_{1}(1+\alpha)}{H_{2}}}}\right) d z \\
\leq & C(t-r)^{-\frac{3 \theta_{1}}{2 H_{2}}-\frac{1-\theta_{1}}{2 H_{2}}}\left((t-r)^{\frac{1}{2}}+(t-r)^{\frac{1}{2}+\frac{\theta_{1}}{H_{2}}}+(t-r)^{\frac{3}{2(1+\alpha)}}\right) \\
\leq & C(t-r)^{-\frac{3 \theta_{1}}{2 H_{2}}-\frac{1-\theta_{1}}{2 H_{2}}+\frac{1}{2}}=C(t-r)^{\frac{1}{2}-\frac{1+2 \theta_{1}}{2 H_{2}}}
\end{aligned}
$$

for all $t>r>0$ and $x \in \mathbb{R}$. Thus, we have introduced (4.1) and hence (4.2) follows.

Lemma 4.2 For all $t>r \geq 0,0<\theta_{2}<H_{2}$, and $x, z \in \mathbb{R}$, we have

$$
\int_{\mathbb{R}}\left(\left|\frac{\partial G_{\alpha}}{\partial x}(r, z ; t, x)\right|^{\theta_{2}} \cdot\left|G_{\alpha}(r, z ; t, x)\right|^{1-\theta_{2}}\right)^{\frac{1}{H_{2}}} d z \leq C(t-r)^{\frac{1}{2}-\frac{1+\theta_{2}}{2 H_{2}}}
$$

and

$$
\int_{0}^{T}\left(\int_{\mathbb{R}}\left(\left|\frac{\partial G_{\alpha}}{\partial x}(r, z ; t, x)\right|^{\theta_{2}}\left|G_{\alpha}(r, z ; t, x)\right|^{1-\theta_{2}}\right)^{\frac{1}{H_{2}}} d z\right)^{\frac{H_{2}}{H_{1}}} d r \leq C .
$$

Proof Given $t>r$ and $z \in \mathbb{R}$. Recall that $\mathbb{D}_{z}=\left\{x \in \mathbb{R}|| x-z \mid<(t-r)^{\frac{3}{2(1+\alpha)}}\right\}$. Then we have

$$
\begin{gathered}
\int_{\mathbb{R}}\left(\left|\frac{\partial G_{\alpha}}{\partial x}(r, z ; t, x)\right|^{\theta_{2}} \cdot\left|G_{\alpha}(r, z ; t, x)\right|^{1-\theta_{2}}\right)^{\frac{1}{H_{2}}} d z \\
\quad \leq \int_{\mathbb{D}_{z}}\left|\frac{\partial G_{1, \alpha}}{\partial x}(r, z ; t, x)\right|^{\frac{\theta_{2}}{H_{2}}}\left|G_{1, \alpha}(r, z ; t, x)\right|^{\frac{1-\theta_{2}}{H_{2}}} d z
\end{gathered}
$$




$$
\begin{aligned}
& +\int_{\overline{\mathbb{D}}_{z}}\left|\frac{\partial G_{2, \alpha}}{\partial x}(r, z ; t, x)\right|^{\frac{\theta_{2}}{H_{2}}}\left|G_{2, \alpha}(r, z ; t, x)\right|^{\frac{1-\theta_{2}}{H_{2}}} d z \\
\equiv & A_{2,1,1}^{2}(t, r, x)+A_{2,1,2}^{2}(t, r, x) .
\end{aligned}
$$

Clearly, we have

$$
\begin{aligned}
A_{2,1,1}^{2}(t, r, x)= & C \int_{\mathbb{D}_{z}}\left|(t-r)^{-1} e^{-\frac{\left.C|x-z|\right|^{2}}{t-r}} \times \frac{2|x-z|}{\sqrt{t-r}}\right|^{\frac{\theta_{2}}{H_{2}}} \\
& \cdot\left|(t-r)^{-\frac{1}{2}} e^{-\frac{C|x-z|^{2}}{t-r}}+(t-r)^{-\frac{1}{2}}\right|^{\frac{1-\theta_{2}}{H_{2}}} d z \\
\leq & C(t-r)^{\frac{3}{2(1+\alpha)}-\frac{1+\theta_{2}}{2 H_{2}}}
\end{aligned}
$$

by the fact $|x| e^{-x^{2}} \leq C$ for all $x \in \mathbb{R}$, and

$$
\begin{aligned}
& A_{2,1,2}^{2}(t, r, x)=\int_{\overline{\mathbb{D}}_{z}}\left|(t-r)^{-\frac{1}{2}} e^{-\frac{C|x-z|^{2}}{t-r}}\left(-\frac{2 C|x-z|}{t-r}\right)-(1+\alpha) \frac{t-r}{|x-z|^{2+\alpha}}\right|^{\frac{\theta_{2}}{H_{2}}} \\
& \cdot\left|(t-r)^{-\frac{1}{2}} e^{-\frac{C|x-z|^{2}}{t-r}}+\frac{t-r}{|x-z|^{1+\alpha}}\right|^{\frac{1-\theta_{2}}{H_{2}}} d z \\
& \leq C(t-r)^{-\frac{3 \theta_{2}}{2 H_{2}}-\frac{1-\theta_{2}}{2 H_{2}}} \int_{\overline{\mathbb{D}}_{z}}|| x-z\left|e^{-\frac{C|x-z|^{2}}{t-r}}+\frac{(t-r)^{5 / 2}}{|x-z|^{2+\alpha}}\right|^{\frac{\theta_{2}}{H_{2}}} \\
& \cdot\left|e^{-\frac{C|x-z|^{2}}{t-r}}+\frac{(t-r)^{3 / 2}}{|x-z|^{1+\alpha}}\right|^{\frac{1-\theta_{2}}{H_{2}}} d z \\
& \leq C(t-r)^{-\frac{3 \theta_{2}}{2 H_{2}}-\frac{1-\theta_{2}}{2 H_{2}}}\left((t-r)^{\frac{\theta_{2}}{2 H_{2}}+\frac{1}{2}}+(t-r)^{\frac{5 \theta_{2}}{2 H_{2}}+\frac{3}{2(1+\alpha)}-\frac{3 \theta_{2}(2+\alpha)}{2 H_{2}(1+\alpha)}}\right) \\
& \leq C(t-r)^{\frac{1}{2}-\frac{1+\theta_{2}}{2 H_{2}}}
\end{aligned}
$$

for all $0<\theta_{2}<H_{2}$. Thus, we have proved the estimate (4.3) and (4.4).

Proof of Theorem 4.1 We shall divide the proof into two steps.

Step 1. We first consider the temporal case. Denote

$$
\begin{aligned}
A_{1}^{1}(t, s, x):= & \int_{\mathbb{R}}\left[G_{\alpha}(0, y ; t, x)-G_{\alpha}(0, y ; s, x)\right] \vartheta(y) d y, \\
A_{2}^{1}(t, s, x):= & \int_{0}^{t} \int_{\mathbb{R}} G_{\alpha}(r, y ; t, x) W(d y, d r)-\int_{0}^{s} \int_{\mathbb{R}} G_{\alpha}(r, y ; s, x) W(d y, d r), \\
A_{3}^{1}(t, s, x):= & \int_{0}^{t} \int_{\mathbb{R}} \frac{\partial G_{\alpha}}{\partial y}(r, y ; t, x) f(r, y, u(r, y)) d y d r \\
& -\int_{0}^{s} \int_{\mathbb{R}} \frac{\partial G_{\alpha}}{\partial y} G_{\alpha}(r, y ; t, x) f(r, y, u(r, y)) d y d r
\end{aligned}
$$

for all $x \in \mathbb{R}$ and $0 \leq s<t \leq T$. Then we have

$$
|u(t, x)-u(s, x)| \leq\left|A_{1}^{1}(t, s, x)\right|+\left|A_{2}^{1}(t, s, x)\right|+\left|A_{3}^{1}(t, s, x)\right|
$$


for all $x \in \mathbb{R}$ and $0 \leq s<t \leq T$. By Hölder's inequality, the semigroup property and (3.2), we have

$$
\begin{aligned}
& E\left|A_{1}^{1}(t, s, x)\right|^{p} \\
& \quad=E\left|\int_{\mathbb{R}^{2}}\left[G_{\alpha}(0, z ; t-s, y) G_{\alpha}(0, y ; s, x)\right] \vartheta(z) d y d z-\int_{\mathbb{R}} G_{\alpha}(0, y ; s, x) \vartheta(y) d y\right|^{p} \\
& \quad=E\left|\int_{\mathbb{R}} G_{\alpha}(0, z ; t-s, 0) \int_{\mathbb{R}} G_{\alpha}(0, y ; s, x)(\vartheta(y-z)-\vartheta(y)) d y d z\right|^{p} \\
& \quad \leq C \int_{\mathbb{R}}\left|G_{\alpha}(0, z ; t-s, 0)\right| \int_{\mathbb{R}} G_{\alpha}(0, y ; s, x) E|\vartheta(y-z)-\vartheta(y)|^{p} d y d z \\
& \quad \leq C \int_{\mathbb{R}}\left|G_{\alpha}(0, y ; s, x)\right| d y \int_{\mathbb{R}}\left|G_{\alpha}(0, z ; t-s, 0)\right||z|^{p \theta} d z
\end{aligned}
$$

with $p \theta<\alpha$. Some elementary calculations can show that

$$
\begin{aligned}
\int_{\mathbb{R}}\left|G_{\alpha}(0, y ; s, x)\right| d y & \leq C\left(\int_{\mathbb{R}} s^{-\frac{1}{2}} e^{-\frac{|x-y|^{2}}{C_{2}}} d y+\int_{\mathbb{R}} s^{-\frac{1}{2}} \wedge \frac{s}{|x-y|^{1+\alpha}} d y\right) \\
& \leq C+C \int_{0}^{\infty} s^{-\frac{1}{2}} \wedge \frac{s}{y^{1+\alpha}} d y \leq C+C s^{-\frac{1}{2}+\frac{3}{2(1+\alpha)}}
\end{aligned}
$$

and

$$
\begin{aligned}
\int_{\mathbb{R}}\left|G_{\alpha}(0, z ; t-s, 0)\right||z|^{p \theta} d z= & C \int_{\mathbb{R}}(t-s)^{-\frac{1}{2}} e^{-\frac{z^{2}}{C_{2}(t-s)}}|z|^{p \theta} d z \\
& +C \int_{\mathbb{R}}\left((t-s)^{-\frac{1}{2}} \wedge \frac{t-s}{|z|^{1+\alpha}}\right)|z|^{p \theta} d z \\
\leq & C(t-s)^{\frac{p \theta}{2}}+C(t-s)^{-\frac{1}{2}+\frac{3(1+p \theta)}{2(1+\alpha)}}
\end{aligned}
$$

which gives

$$
E\left|A_{1}^{1}(t, s, x)\right|^{p} \leq C(t-s)^{\frac{p \theta}{2}} .
$$

Let now us estimate the term $A_{2}^{1}(t, s, x)$. Denote

$$
\begin{aligned}
& A_{2,1}^{1}(t, s, x):=\int_{0}^{s} \int_{\mathbb{R}}\left(G_{\alpha}(r, z ; t, x)-G_{\alpha}(r, z ; s, x)\right) W(d z, d r), \\
& A_{2,2}^{1}(t, s, x):=\int_{s}^{t} \int_{\mathbb{R}} G_{\alpha}(r, z ; t, x) W(d z, d r)
\end{aligned}
$$

for all $x \in \mathbb{R}$ and $0 \leq s<t \leq T$. We then have

$$
\left|A_{2}^{1}(t, s, x)\right| \leq\left|A_{2,1}^{1}(t, s, x)\right|+\left|A_{2,2}^{1}(t, s, x)\right|
$$

for all $x \in \mathbb{R}$ and $0 \leq s<t \leq T$. Moreover, for every $\theta_{1} \in(0,1)$ we let

$$
\begin{aligned}
& A_{2,1,1}^{1}(t, s, x):=\left\|\left|G_{\alpha}(\cdot, \cdot ; t, x)-G_{\alpha}(\cdot, \cdot ; s, x)\right|^{\theta_{1}}\left|G_{\alpha}(\cdot, \cdot ; t, x)\right|^{1-\theta_{1}}\right\|_{\mathcal{H}^{\prime}}^{2} \\
& A_{2,1,2}^{1}(t, s, x):=\left\|\left|G_{\alpha}(\cdot, \cdot ; t, x)-G_{\alpha}(\cdot, \cdot ; s, x)\right|^{\theta_{1}}\left|G_{\alpha}(\cdot, \cdot ; s, x)\right|^{1-\theta_{1}}\right\|_{\mathcal{H}}^{2}
\end{aligned}
$$


with $x \in \mathbb{R}$ and $0 \leq s<t \leq T$. Then we have

$$
\begin{aligned}
E\left|A_{2,1}^{1}(t, s, x)\right|^{p} & \leq C\left\|G_{\alpha}(\cdot, \cdot ; t, x)-G_{\alpha}(\cdot, \cdot ; s, x)\right\|_{\mathcal{H}}^{p} \\
& =C\left(\left\|\left|G_{\alpha}(\cdot, \cdot ; t, x)-G_{\alpha}(\cdot, \cdot ; s, x)\right|^{\theta_{1}}\left|G_{\alpha}(\cdot, \cdot ; t, x)-G_{\alpha}(\cdot, \cdot ; s, x)\right|^{1-\theta_{1}}\right\|_{\mathcal{H}}^{2}\right)^{\frac{p}{2}} \\
& \leq C\left(\left|A_{2,1,1}^{1}(t, s, x)\right|+\left|A_{2,1,2}^{1}(t, s, x)\right|\right)^{\frac{p}{2}}
\end{aligned}
$$

for all $x \in \mathbb{R}$ and $0 \leq s<t \leq T$. But, by using (3.2), Proposition 2.4, Lemma 4.1, and the mean-value theorem, we see that there is an $\xi$ between $s$ and $t$ such that

$$
\begin{aligned}
\left|A_{2,1,1}^{1}(t, s, x)\right| & ||\left|\frac{\partial G_{\alpha}}{\partial t}(\cdot, \cdot ; \xi, x)\right|^{\theta_{1}}|t-s|^{\theta_{1}}\left|G_{\alpha}(\cdot, \cdot ; t, x)\right|^{1-\theta_{1}} \|_{\mathcal{H}}^{2} \\
= & |t-s|^{2 \theta_{1}} \int_{0}^{t} \int_{0}^{t} d r_{1} d r_{2} \int_{\mathbb{R}} \int_{\mathbb{R}}\left|\frac{\partial G_{\alpha}}{\partial t}\left(r_{1}, z_{1} ; \xi, x\right)\right|^{\theta_{1}}\left|G_{\alpha}\left(r_{1}, z_{1} ; t, x\right)\right|^{1-\theta_{1}} \\
& \cdot \Xi_{H}\left(r_{1}, r_{2} ; z_{1}, z_{2}\right)\left|\frac{\partial G_{\alpha}}{\partial t}\left(r_{2}, z_{2} ; \xi, x\right)\right|^{\theta_{1}}\left|G_{\alpha}\left(r_{2}, z_{2} ; t, x\right)\right|^{1-\theta_{1}} d z_{1} d z_{2} \\
\leq & C|t-s|^{2 \theta_{1}}\left(\int_{0}^{T}\left(\int_{\mathbb{R}}\left(\left|\frac{\partial G_{\alpha}}{\partial t}(r, z ; t, x)\right|^{\theta_{1}}\left|G_{\alpha}(r, z ; t, x)\right|^{1-\theta_{1}}\right)^{\frac{1}{H_{2}}} d z\right)^{\frac{H_{2}}{H_{1}}} d r\right)^{2 H_{1}} \\
\leq & C|t-s|^{2 \theta_{1}}
\end{aligned}
$$

for all $0<\theta_{1}<\frac{2 H_{1}-1}{2}+\frac{H_{2}}{2}$. Similarly, one can prove that

$$
\left|A_{2,1,2}^{1}(t, s, x)\right| \leq C|t-s|^{2 \theta_{1}} .
$$

It follows that

$$
E\left|A_{2,1}^{1}(t, s, x)\right|^{p} \leq C|t-s|^{p \theta_{1}}
$$

for all $\theta_{1} \in\left(0, \vartheta_{1}\right)$. On the other hand, we have

$$
\begin{aligned}
\int_{s}^{t} & \int_{s}^{t} d r_{1} d r_{2} \int_{\mathbb{R}^{2}} G_{\alpha}\left(r_{1}, z_{1} ; t, x\right) \Xi\left(r_{1}, r_{2} ; z_{1}, z_{2}\right) G_{\alpha}\left(r_{2}, z_{2} ; t, x\right) d z_{1} d z_{2} \\
= & \int_{s}^{t} \int_{s}^{t}\left|r_{1}-r_{2}\right|^{2 H_{1}-2} d r_{1} d r_{2} \int_{\mathbb{R}^{2}}\left|z_{1}-z_{2}\right|^{2 H_{2}-2} \\
& \cdot G_{\alpha}\left(r_{1}, z_{1} ; t, x\right) G_{\alpha}\left(r_{2}, z_{2} ; t, x\right) d z_{1} d z_{2} d r_{1} d r_{2} \\
\leq & C \int_{s}^{t} \int_{s}^{t}\left|r_{1}-r_{2}\right|^{2 H_{1}-2}\left\|G_{\alpha}\left(r_{1}, \cdot ; t, x\right)\right\|_{L^{\frac{1}{H_{2}}}(\mathbb{R})}\left\|G_{\alpha}\left(r_{2}, \cdot ; t, x\right)\right\|_{L^{\frac{1}{H_{2}}(\mathbb{R})}} d r_{1} d r_{2} \\
\leq & C\left(\int_{s}^{t}\left(\left\|G_{\alpha}(r, \cdot ; t, x)\right\|_{L^{\frac{1}{H_{2}}}(\mathbb{R})}\right)^{\frac{1}{H_{1}}} d r\right)^{2 H_{1}} \leq C|t-s|^{\left(2 H_{1}+H_{2}-1\right)}
\end{aligned}
$$

for all $x \in \mathbb{R}$ and $0 \leq s<t \leq T$, which gives

$$
E\left|A_{2,2}^{1}(t, s, x)\right|^{p}=E\left|\int_{s}^{t} \int_{\mathbb{R}} G_{\alpha}(r, z ; t, x) W(d r, d z)\right|^{p} \leq C|t-s|^{\frac{p}{2}\left(2 H_{1}+H_{2}-1\right)}
$$


for all $x \in \mathbb{R}$ and $0 \leq s<t \leq T$ by (3.7). Combining this with (4.6), we get

$$
E\left|A_{2}^{1}(t, s, x)\right|^{p} \leq C|t-s|^{p \theta_{1}}
$$

for $\theta_{1} \in\left(0, \vartheta_{1}\right)$.

Finally, by the Hölder inequality, Assumption 2, Theorem 3.1, and (3.8), we have

$$
\begin{aligned}
E\left(\left|A_{3,1}^{1}(t, s, x)\right|^{p}\right) & :=E\left|\int_{0}^{s} d r \int_{\mathbb{R}} \frac{\partial G_{\alpha}}{\partial y}(r, y ; s, x)[f(r+t-s, y, u(r+t-s, y))-f(r, y, u(r, y))] d y\right|^{p} \\
\leq & C\left(\int_{0}^{s} d r \int_{\mathbb{R}} \frac{\partial G_{\alpha}}{\partial y}(r, y ; s, x) d y\right)^{p-1} \int_{0}^{s} d r \int_{\mathbb{R}} \frac{\partial G_{\alpha}}{\partial y}(r, y ; s, x) \\
& \cdot E|f(r+t-s, y, u(r+t-s, y))-f(r, y, u(r, y))|^{p} d y \\
\leq & C\left(|t-s|^{p}+\int_{0}^{s} \sup _{y \in \mathbb{R}} E|u(r+t-s, y)-u(r, y)|^{p} d r\right)
\end{aligned}
$$

and

$$
\begin{aligned}
E\left(\left|A_{3,2}^{1}(t, s, x)\right|^{p}\right):= & \left|\int_{0}^{t-s} d r \int_{\mathbb{R}} \frac{\partial G_{\alpha}}{\partial y}(r, y ; t, x) f(r, y, u(r, y)) d y\right|^{p} \\
\leq & C\left(\int_{0}^{t-s} d r \int_{\mathbb{R}} \frac{\partial G_{\alpha}}{\partial y}(r, y ; s, x) d y\right)^{p-1} \\
& \times \int_{0}^{t-s} d r \int_{\mathbb{R}} \frac{\partial G_{\alpha}}{\partial y}(r, y ; t, x) E|f(r, y, u(r, y))|^{p} d y \\
\leq & C|t-s|^{p}\left(1+\sup _{(t, x) \in[0, T] \times \mathbb{R}} E|u(t, x)|^{p}\right) \leq C|t-s|^{p}
\end{aligned}
$$

for all $x \in \mathbb{R}$ and $0 \leq s<t \leq T$. It follows that

$$
\begin{aligned}
E\left|A_{3}^{1}(t, s, x)\right|^{p}= & E \mid \int_{0}^{t} \int_{\mathbb{R}} \frac{\partial G_{\alpha}}{\partial y}(r, y ; t, x) f(r, y, u(r, y)) d y d r \\
& -\left.\int_{0}^{s} \int_{\mathbb{R}} \frac{\partial G_{\alpha}}{\partial y} G_{\alpha}(r, y ; t, x) f(r, y, u(r, y)) d y d r\right|^{p} \\
\leq & C\left(E\left|A_{3,1}^{1}(t, s, x)\right|^{p}+E\left|A_{3,2}^{1}(t, s, x)\right|^{p}\right) \\
\leq & C\left(|t-s|^{p}+\int_{0}^{s} \sup _{y \in \mathbb{R}} E|u(r+t-s, y)-u(r, y)|^{p} d r\right)
\end{aligned}
$$

for all $x \in \mathbb{R}$ and $0 \leq s<t \leq T$.

Thus, we have obtained the desired estimate

$$
\begin{aligned}
E|u(t, x)-u(s, x)|^{p} \leq & C\left(E\left|A_{1}^{1}(t, s, x)\right|^{p}+E\left|A_{2}^{1}(t, s, x)\right|^{p}+E\left|A_{3}^{1}(t, s, x)\right|^{p}\right) \\
\leq & C\left(|t-s|^{p \theta}+|t-s|^{p \theta_{1}}\right. \\
& \left.+|t-s|^{p}+\int_{0}^{s} \sup _{y \in \mathbb{R}} E|u(r+t-s, y)-u(r, y)|^{p} d r\right)
\end{aligned}
$$


for all $x \in \mathbb{R}$ and $0 \leq s<t \leq T$, which implies that

$$
E|u(t, x)-u(s, x)|^{p} \leq C\left(|t-s|^{p v}+\int_{0}^{s} \sup _{y \in \mathbb{R}} E|u(r+t-s, y)-u(r, y)|^{p} d r\right)
$$

for all $x \in \mathbb{R}$ and $0 \leq s<t \leq T$ by taking $v \in \min \left\{\theta, \theta_{1}\right\}$. This shows the Hölder continuity in time variables $t$ by Gronwall's inequality.

Step 2. We consider the spatial case. For all $t \in[0, T]$ and $x, y \in \mathbb{R}$, we need to estimate the following expressions:

$$
\begin{aligned}
& A_{1}^{2}(t, x, y):=\left|\int_{\mathbb{R}}\left[G_{\alpha}(0, z ; t, x)-G_{\alpha}(0, z ; t, y)\right] \vartheta(z) d z\right|, \\
& A_{2}^{2}(t, x, y):=\left|\int_{0}^{t} \int_{\mathbb{R}}\left[G_{\alpha}(r, z ; t, x)-G_{\alpha}(r, z ; t, y)\right] W(d z, d r)\right| \\
& A_{3}^{2}(t, x, y):=\left|\int_{0}^{t} \int_{\mathbb{R}}\left[\frac{\partial G_{\alpha}}{\partial z}(r, z ; t, x)-\frac{\partial G_{\alpha}}{\partial z}(r, z ; t, y)\right] f(r, z, u(r, z)) d z d r\right| .
\end{aligned}
$$

We have

$$
E\left|A_{1}^{2}(t, x, y)\right|^{p} \leq \sup _{z \in \mathbb{R}} E|\vartheta(z+x-y)-\vartheta(z)|^{p} \cdot\left|\int_{\mathbb{R}} G_{\alpha}(0, z ; t, x) d z\right|^{p} \leq|x-y|^{p \theta}
$$

for all $t \in[0, T]$ and $x, y \in \mathbb{R}$ by Assumption 1 . Denote

$$
\begin{aligned}
& A_{2,1}^{2}(t, x, y):=\left\|\left|G_{\alpha}(\cdot, \cdot ; t, x)-G_{\alpha}(\cdot, \cdot ; t, y)\right|^{\theta_{2}}\left|G_{\alpha}(\cdot, \cdot ; t, x)\right|^{1-\theta_{2}}\right\|_{\mathcal{H}}, \\
& A_{2,2}^{2}(t, x, y):=\left\|\left|G_{\alpha}(\cdot, \cdot ; t, x)-G_{\alpha}(\cdot, \cdot ; t, y)\right|^{\theta_{2}}\left|G_{\alpha}(\cdot, \cdot ; t, y)\right|^{1-\theta_{2}}\right\|_{\mathcal{H}}
\end{aligned}
$$

for all $t \geq 0$ and $x, y \in \mathbb{R}$. Then we have

$$
\begin{aligned}
E\left|A_{2}^{2}(t, x, y)\right|^{p} & =C\left\|\left|G_{\alpha}(\cdot, \cdot ; t, x)-G_{\alpha}(\cdot, \cdot ; t, y)\right|^{\theta_{2}}\left|G_{\alpha}(\cdot, \cdot ; t, x)-G_{\alpha}(\cdot, \cdot ; t, y)\right|^{1-\theta_{2}}\right\|_{\mathcal{H}}^{p} \\
& \leq C\left(A_{2,1}^{2}(t, x, y)+A_{2,2}^{2}(t, x, y)\right)^{p} .
\end{aligned}
$$

Similar to Step I, by using (3.2), Proposition 2.4, Lemma 4.2, and the mean-value theorem, one can see that

$$
\begin{aligned}
A_{2,1}^{2}(t, x, y)= & \left\|\left|\frac{\partial G_{\alpha}}{\partial x}(\cdot, \cdot ; t, \xi)\right|^{\theta_{2}}|x-y|^{\theta_{2}}\left|G_{\alpha}(\cdot, \cdot ; t, x)\right|^{1-\theta_{2}}\right\|_{\mathcal{H}} \\
\leq & C|x-y|^{\theta_{2}}\left(\int_{0}^{T} \int_{0}^{T} \int_{\mathbb{R}^{2}}\left|\frac{\partial G_{\alpha}}{\partial x}\left(r_{1}, z_{1} ; t, \xi\right)\right|^{\theta_{2}}\left|G_{\alpha}\left(r_{1}, z_{1} ; t, x\right)\right|^{1-\theta_{2}}\right. \\
& \times \Xi_{H}\left(r_{1}, r_{2} ; z_{1}, z_{2}\right) \\
& \left.\cdot\left|\frac{\partial G_{\alpha}}{\partial x}\left(r_{2}, z_{2} ; t, \xi\right)\right|^{\theta_{2}}\left|G_{\alpha}\left(r_{2}, z_{2} ; t, x\right)\right|^{1-\theta_{2}} d z_{1} d z_{2} d r_{1} d r_{2}\right)^{\frac{1}{2}} \\
\leq & C|x-y|^{\theta_{2}}\left(\int_{0}^{T}\left(\int_{\mathbb{R}}\left(\left|\frac{\partial G_{\alpha}}{\partial x}(r, z ; t, x)\right|^{\theta_{2}}\left|G_{\alpha}(r, z ; t, x)\right|^{1-\theta_{2}}\right)^{\frac{1}{H_{2}}} d z\right)^{\frac{H_{2}}{H_{1}}} d r\right)^{H_{1}} \\
\leq & C|x-y|^{\theta_{2}}
\end{aligned}
$$


for all $\theta_{2}<H_{2}$. Similarly, one can also prove

$$
A_{2,2}^{2}(t, x, y) \leq C|x-y|^{\theta_{2}}
$$

for all $\theta_{2}<H_{2}$. It follows that

$$
E\left|A_{2}^{2}(t, x, y)\right|^{p} \leq C|x-y|^{p \theta_{2}}
$$

for all $\theta_{2}<H_{2}$. Finally, we consider the term $\left|A_{3}^{2}(t, x, y)\right|$. By the Hölder inequality, Assumption 1 , and (3.8), we have

$$
\begin{aligned}
& E\left|A_{3}^{2}(t, x, y)\right|^{p} \\
& \quad=E\left|\int_{0}^{t} d r \int_{\mathbb{R}}\left[\frac{\partial G_{\alpha}}{\partial z}(r, z ; t, x)-\frac{\partial G_{\alpha}}{\partial z}(r, z ; t, y)\right] f(r, z, u(r, z)) d z\right|^{p} \\
& \quad \leq C \int_{0}^{t} d r \int_{\mathbb{R}}\left|\frac{\partial G_{\alpha}}{\partial z}(r, z ; t, y)\right| E|f(r, z+x-y, u(r, z+x-y))-f(r, z, u(r, z))|^{p} d z \\
& \quad \leq C \int_{\mathbb{R}} d z\left|\frac{\partial G_{\alpha}}{\partial z}(r, z ; t, y)\right| \int_{0}^{t}\left(|x-y|^{p}+\sup _{z \in \mathbb{R}} E|u(r, z+x-y)-u(r, z)|^{p}\right) d r \\
& \quad \leq C\left(t|x-y|^{p}+\int_{0}^{t} \sup _{z \in \mathbb{R}} E|u(r, z+x-y)-u(r, z)|^{p} d r\right) .
\end{aligned}
$$

Combining this with (4.13) and (4.14), we have

$$
\begin{aligned}
E|u(t, x)-u(t, y)|^{p} & \leq C\left(E\left|A_{1}^{2}(t, x, y)\right|^{p}+E\left|A_{2}^{2}(t, x, y)\right|^{p}+E\left|A_{3}^{2}(t, x, y)\right|^{p}\right) \\
& \leq C\left(|x-y|^{p \theta_{2}}+t|x-y|^{p}+\int_{0}^{t} \sup _{z \in \mathbb{R}} E|u(r, z+x-y)-u(r, z)|^{p} d r\right)
\end{aligned}
$$

for all $0<\theta_{2}<H_{2}$. Thus, we have proved the Hölder continuity in space variables $x$ by Gronwall's inequality.

As an immediate result of the above theorem, we see that the quadratic variation is zero. At the end of this section, we give the $p$-variation of the solution. For convenience we consider the following special equation:

$$
\left\{\begin{array}{l}
\frac{\partial \bar{u}}{\partial t}=\Delta_{\alpha} \bar{u}+\frac{\partial^{2} W}{\partial t \partial x}, \\
\bar{u}(0, x)=0, \quad x \in \mathbb{R} .
\end{array}\right.
$$

As in Section 3, the solution of (4.16) can be written in mild form as

$$
\bar{u}(t, x)=\int_{0}^{t} \int_{\mathbb{R}} \bar{G}_{\alpha}(s, y ; t, x) W(d s, d y),
$$

where $\bar{G}_{\alpha}(s, y ; t, x)$ stands for the heat kernel of $\Delta_{\alpha}$. It follows from Chen et al. [23] that

$$
\bar{G}_{\alpha}(s, y ; t, x) \leq C\left((t-s)^{-\frac{1}{\alpha}} \wedge \frac{t-s}{|x-y|^{1+\alpha}}\right)
$$

for all $0 \leq s<t, x, y \in \mathbb{R}$ and some constant $C \geq 0$. 
Lemma 4.3 Let $\bar{u}$ be the solution of (4.16). Then, for all $0<s<t$, we have

$$
E|\bar{u}(s, x)-\bar{u}(t, x)|^{2} \leq C|t-s|^{2 \eta}
$$

where $\eta \in\left(0, \frac{\alpha H_{1}+H_{2}-1}{\alpha}\right)$.

Proof Similar to the proof of Step 1 of Theorem 4.1 and Lemma 4.1, when $\eta \in\left(0, \frac{\alpha H_{1}+H_{2}-1}{\alpha}\right)$, we obtain

$$
\begin{aligned}
E|\bar{u}(t, x)-\bar{u}(s, x)|^{2}= & E\left|\int_{0}^{t} \int_{\mathbb{R}} \bar{G}_{\alpha}(r, y ; t, x) W(d y, d r)-\int_{0}^{s} \int_{\mathbb{R}} \bar{G}_{\alpha}(r, y ; s, x) W(d y, d r)\right|^{2} \\
\leq & E\left|\int_{0}^{s} \int_{\mathbb{R}}\left(\bar{G}_{\alpha}(r, z ; t, x)-\bar{G}_{\alpha}(r, z ; s, x)\right) W(d z, d r)\right|^{2} \\
& +E\left|\int_{s}^{t} \int_{\mathbb{R}} \bar{G}_{\alpha}(r, z ; t, x) W(d z, d r)\right|^{2} \\
\leq & C_{p, \eta}\left(\left\|\left|\bar{G}_{\alpha}(\cdot, \cdot ; t, x)-\bar{G}_{\alpha}(\cdot, \cdot ; s, x)\right|^{\eta} \cdot\left|\bar{G}_{\alpha}(\cdot, \cdot ; t, x)\right|^{1-\eta}\right\|_{\mathcal{H}}^{2}\right. \\
& \left.+\left\|\left|\bar{G}_{\alpha}(\cdot, \cdot ; t, x)-\bar{G}_{\alpha}(\cdot, \cdot ; s, x)\right|^{\eta} \cdot\left|\bar{G}_{\alpha}(\cdot, \cdot ; s, x)\right|^{1-\eta}\right\|_{\mathcal{H}}^{2}\right) \\
& +C\left(\int_{0}^{t}\left(\left\|\bar{G}_{\alpha}(r, \cdot ; t, x)\right\|_{L^{\frac{1}{H_{2}}(\mathbb{R})}}\right)^{\frac{1}{H_{1}}} d s\right)^{2 H_{1}} \\
\leq & C|t-s|^{2 \eta} .
\end{aligned}
$$

This completes the proof.

For $T>0$, let $\tau_{n}=\left\{0=t_{0}<t_{1}<\cdots<t_{n}=T\right\}$ be a partition of $[0, T]$ such that the mesh size $\left|\Delta_{n}\right|=\max _{j}\left|t_{j}-t_{j-1}\right| \rightarrow 0(n \rightarrow \infty)$. Recall that a process $Y=\left\{Y_{t} ; 0 \leq t<\infty\right\}$ is of bounded $p$-variation with $p \geq 1$ on the interval $[0, T]$ if the limit of

$$
V_{n}^{p}(Y ; T):=\sum_{j=0}^{n-1}\left|Y_{t_{j+1}}-Y_{t_{j}}\right|^{p},
$$

exists in $L^{1}(\Omega)$, as $n \rightarrow \infty$, We denote by $V^{p}(Y ; T)$ the $p$-variation on $[0, T]$.

We new consider $p$-variations of the solution to the fractional heat equation (4.16).

Theorem 4.2 Let $(\bar{u}(t, x), t \in[0, T], x \in \mathbb{R})$ be given by (4.17). For $H_{1} \in\left(\frac{1}{2}, 1\right)$, and $H_{2}=\frac{1}{2}$, we have

$$
V^{p}(\bar{u}(\cdot, x) ; T)=0
$$

if $p>\frac{2 \alpha}{2 \alpha H_{1}-1}$ for all $x \in \mathbb{R}$.

Proof By Lemma 4.3, we have

$$
\begin{aligned}
E\left(V^{p}(\bar{u}(t, x) ; T)\right) & =E\left(\sum_{i=0}^{n-1}\left|\bar{u}\left(t_{i+1}, x\right)-\bar{u}\left(t_{i}, x\right)\right|^{p}\right) \\
& =\sum_{i=0}^{n-1} E\left|\bar{u}\left(t_{i+1}, x\right)-\bar{u}\left(t_{i}, x\right)\right|^{p}
\end{aligned}
$$




$$
\begin{aligned}
& \leq \sum_{i=0}^{n-1}\left(E\left|\bar{u}\left(t_{i+1}, x\right)-\bar{u}\left(t_{i}, x\right)\right|^{2}\right)^{\frac{p}{2}} \\
& \leq C \sum_{i=0}^{n-1}\left|t_{i+1}-t_{i}\right|^{\frac{p\left(2 \alpha H_{1}-1\right)}{2 \alpha}},
\end{aligned}
$$

which shows that $t \mapsto \bar{u}(t, x)$ has $p$-variation 0 when $p>\frac{2 \alpha}{2 \alpha H_{1}-1}$ for all $x \in \mathbb{R}$. This completes the proof.

\section{Existence of the density}

In this part, we will focus to prove the absolute continuity of the distribution of solution $\{u(t, x):(t, x) \in[0, T] \times \mathbb{R}\}$ given in Section 3 by using Malliavin calculus.

Proposition 5.1 Under the assumptions in Theorem 3.1, if the function $(t, x, z) \mapsto f(t, x, z)$ and its partial derivatives of order 1 are bounded, then $u(t, x) \in \mathbb{D}^{1,2}$ and

$$
D_{r, v} u(t, x)=G_{\alpha}(r, v ; t, x)+\int_{\tau}^{t} \int_{\mathbb{R}} \frac{\partial G_{\alpha}}{\partial y}(s, y ; t, x) \frac{\partial f}{\partial z}(s, y, u(s, y)) D_{r, v} u(s, y) d y d s
$$

for all $0 \leq r \leq t \leq T$ and $x, v \in \mathbb{R}$.

Proof By approximating we can introduce the theorem. Let $u_{n}(t, x)$ satisfy the next equation:

$$
\begin{aligned}
u_{0}(t, x)= & \int_{\mathbb{R}} G_{\alpha}(0, y ; t, x) \vartheta(y) d y, \\
u_{n+1}(t, x)= & u_{0}(t, x)+\int_{0}^{t} \int_{\mathbb{R}} G_{\alpha}(s, y ; t, x) W(d s, d y) \\
& +\int_{0}^{t} \int_{\mathbb{R}} \frac{\partial G_{\alpha}}{\partial y}(s, y ; t, x) f\left(s, y, u_{n}(s, y)\right) d y d s
\end{aligned}
$$

for all $n=0,1,2, \ldots$ Then $u_{n}(t, x) \in \mathbb{D}_{h}$ and it satisfies

$$
\begin{aligned}
D_{h} u_{n}(t, x)= & \left\langle G_{\alpha}(\cdot, \cdot ; t, x), h\right\rangle_{\mathcal{H}}+\int_{0}^{t} \int_{\mathbb{R}} \frac{\partial G_{\alpha}}{\partial y}(s, y ; t, x) \\
& \cdot \frac{\partial f}{\partial z}\left(s, y, u_{n-1}(s, y)\right) D_{h} u_{n-1}(s, y) d y d s .
\end{aligned}
$$

for each $n \in \mathbb{N}$ and $h \in \mathcal{H}$ (see the argument in Zhang and Zheng [28]). Since

$$
\lim _{n \rightarrow+\infty} u_{n}=u
$$

in $L^{p}$, there is a random field $u^{(h)}(t, x)$ such that

$$
\lim _{n \rightarrow+\infty} D_{h} u_{n}(t, x)=u^{(h)}(t, x)
$$

uniformly on $(t, x) \in[0, T] \in \mathbb{R}$, and

$$
u^{(h)}(t, x)=\left\langle G_{\alpha}(\cdot, \cdot ; t, x), h\right\rangle_{\mathcal{H}}+\int_{0}^{t} d s \int_{\mathbb{R}} \frac{\partial G_{\alpha}}{\partial y}(s, y ; t, x) \frac{\partial f}{\partial z}(s, y, u(s, y)) u^{(h)}(s, y) d y .
$$


It follows from the closeness of the operator $D_{h}$ that $D_{h} u(t, x)=u^{(h)}(t, x), u(t, x) \in \mathbb{D}_{h}$, and

$$
\begin{aligned}
D_{h} u(t, x)= & \left\langle G_{\alpha}(\cdot, \cdot ; t, x), h\right\rangle_{\mathcal{H}} \\
& +\int_{0}^{t} d s \int_{\mathbb{R}} \frac{\partial G_{\alpha}}{\partial y}(s, y ; t, x) \frac{\partial f}{\partial z}(s, y, u(s, y)) D_{h} u(s, y) d y
\end{aligned}
$$

for all $0 \leq t \leq T$ and $x \in \mathbb{R}$. Now, we claim that $u(t, x) \in \mathbb{D}^{1,2}$. By (5.2), we have

$$
\begin{aligned}
& E\left|D_{h_{n}} u(t, x)\right|^{2} \\
& \quad=E\left|\left\langle G_{\alpha}(\cdot, \cdot ; t, x), h_{n}\right\rangle_{\mathcal{H}}+\int_{0}^{t} d s \int_{\mathbb{R}} \frac{\partial G_{\alpha}}{\partial y}(s, y ; t, x) \frac{\partial f}{\partial z}(s, y, u(s, y)) D_{h_{n}} u(s, y) d y\right|^{2} \\
& \quad \leq C\left\langle G_{\alpha}(\cdot, \cdot ; t, x),\left.h_{n}\right|_{\mathcal{H}} ^{2}+C \int_{0}^{t} d s \int_{\mathbb{R}}\left(\frac{\partial G_{\alpha}}{\partial y}(s, y ; t, x)\right)^{2} E\left(D_{h_{n}} u(s, y)\right)^{2} d y\right.
\end{aligned}
$$

for all $0 \leq t \leq T, x \in \mathbb{R}$ and $\left\{h_{n}, n \geq 1\right\} \subset \mathcal{H}$. Set

$$
\mathcal{K}_{l}(t)=\sup _{x \in \mathbb{R}} \sum_{n=1}^{l} E\left|D_{h_{n}} u(t, x)\right|^{2}
$$

for all $0 \leq t \leq T$. Then we have

$$
\begin{aligned}
\mathcal{K}_{l}(t) & \leq C E \int_{0}^{t} d s \int_{\mathbb{R}}\left(\frac{\partial G_{\alpha}}{\partial y}(s, y ; t, x)\right)^{2} \mathcal{K}_{l}(s) d y+C\left\|G_{\alpha}(\cdot, \cdot ; t, x)\right\|_{\mathcal{H}}^{2} \\
& \leq C+C \int_{0}^{t}(t-s)^{-\frac{1}{2}-\frac{\alpha}{2}} \mathcal{K}_{l}(s) d s
\end{aligned}
$$

for all $t \in[0, T]$ by (5.3) and Cauchy's inequality, where we have used the fact that

$$
\int_{\mathbb{R}}\left(\frac{\partial G_{\alpha}}{\partial y}(s, y ; t, x)\right)^{2} d y \leq C|t-s|^{-\frac{1}{2}-\frac{\alpha}{2}}
$$

for all $s, t \in[0, T]$ and $x \in \mathbb{R}$. It follows from Gronwall's inequality that

$$
\mathcal{K}_{l}(t) \leq C e^{C T^{1-\frac{1}{2}-\frac{\alpha}{2}}}
$$

Letting $l \rightarrow+\infty$, we get

$$
\sup _{x \in \mathbb{R}} E \sum_{n=1}^{\infty}\left|D_{h_{n}} u(t, x)\right|^{2}<+\infty
$$

which shows that $u(t, x) \in \mathbb{D}^{1,2}$ for all $0 \leq t \leq T$ and $x \in \mathbb{R}$.

Finally, let us calculate the derivative $D u(t, x)$ for all $(t, x) \in[0, T] \times \mathbb{R}$. Since $u(t, x)$ is $\mathscr{F}_{t}$-adapted, by Proposition 2.4 there exists a measurable function $D_{r, v} u(t, x) \in \mathcal{H}$ such that $D_{r, v} u(t, x)=0$ if $r>t$ and for any $h \in \mathcal{H}$,

$$
D_{h} u(t, x)=\langle D u(t, x), h\rangle_{\mathcal{H}}
$$


for all $0 \leq t \leq T$ and $x \in \mathbb{R}$. From (5.2), (5.4), and the Fubini theorem, it follows that

$$
\begin{aligned}
\langle D u(t, x), h\rangle_{\mathcal{H}} & \\
= & \left\langle G_{\alpha}(\cdot, \cdot ; t, x), h\right\rangle_{\mathcal{H}}+\int_{0}^{t} d s \int_{\mathbb{R}} \frac{\partial G_{\alpha}}{\partial y}(s, y ; t, x) \frac{\partial f}{\partial z}(s, y, u(s, y)) D_{h} u(s, y) d y \\
= & \left\langle G_{\alpha}(\cdot, \cdot ; t, x), h\right\rangle_{\mathcal{H}}+\int_{0}^{t} d s \int_{\mathbb{R}} \frac{\partial G_{\alpha}}{\partial y}(s, y ; t, x) \frac{\partial f}{\partial z}(s, y, u(s, y))\langle D u(s, y), h\rangle_{\mathcal{H}} d y \\
= & \left\langle G_{\alpha}(\cdot, \cdot ; t, x), h\right\rangle_{\mathcal{H}}+\int_{0}^{t} d s \int_{\mathbb{R}} \frac{\partial G_{\alpha}}{\partial y}(s, y ; t, x) \frac{\partial f}{\partial z}(s, y, u(s, y)) d y \\
& \cdot \int_{0}^{s} \int_{0}^{s} d r d r^{\prime} \int_{\mathbb{R}^{2}} D_{r, v} u(s, y) h\left(r^{\prime}, z^{\prime}\right) \Xi_{H}\left(r, r^{\prime} ; v^{\prime}, z^{\prime}\right) d v^{\prime} d z^{\prime} \\
= & \left\langle G_{\alpha}(\cdot, \cdot ; t, x), h\right\rangle_{\mathcal{H}}+\int_{0}^{t} \int_{0}^{t} d r d r^{\prime} \int_{\mathbb{R}^{2}} h\left(r^{\prime}, z^{\prime}\right) \Xi_{H}\left(r, r^{\prime} ; v^{\prime}, z^{\prime}\right) d v^{\prime} d z^{\prime} \\
& \cdot \int_{r}^{t} d s \int_{\mathbb{R}} \frac{\partial G_{\alpha}}{\partial y}(s, y ; t, x) \frac{\partial f}{\partial z}(s, y, u(s, y)) d y
\end{aligned}
$$

for $(t, x) \in[0, T] \times \mathbb{R}$. Thus, we have proved the desired formula,

$$
D_{r, v} u(t, x)=G_{\alpha}(r, v ; t, x)+\int_{r}^{t} d s \int_{\mathbb{R}} \frac{\partial G_{\alpha}}{\partial y}(s, y ; t, x) \frac{\partial f}{\partial z}(s, y, u(s, y)) D_{r, v} u(s, y) d y
$$

for all $0 \leq r \leq t, x, v \in \mathbb{R}$, and the theorem follows.

Theorem 5.2 Under the assumptions of Theorem 3.1 and assuming the function $f$ and its partial derivatives of order 1 to be bounded, the distribution of the random variable $u(t, x)$ is absolutely continuous with respect to the Lebesgue measure for all $(t, x) \in[0, T] \times \mathbb{R}$.

For proving Theorem 5.2, we will make use of the following lemma.

Lemma 5.1 Let $t>0$ and $0<r<t$. Denote

$$
\mathcal{J}_{r}(s, y)=\int_{t-r}^{t} d l \int_{\mathbb{R}} E\left|D_{l, v} u(s, y)\right|^{2} d v
$$

for $s \in[t-r, t]$ and $y \in \mathbb{R}$. Then we have

$$
\sup _{(s, y) \in[t-r, t] \times \mathbb{R}} \mathcal{J}_{r}(s, y)<C r^{\frac{\alpha-1}{2}} .
$$

Proof Let $0<r<t$ and $s \in[t-r, t]$. Then we have

$$
\sup _{(s, y) \in[0, t] \times \mathbb{R}} \mathcal{J}_{r}(s, y)<+\infty
$$

by the proof of Proposition 5.1. Denote

$$
\mathcal{J}_{r, 1}(s, y):=\int_{t-r}^{s} \int_{\mathbb{R}}\left|G_{\alpha}(v, z ; s, y)\right|^{2} d z d v,
$$




$$
\begin{aligned}
\mathcal{J}_{r, 2}(s, y):= & \int_{t-r}^{s} d l \int_{\mathbb{R}} d v E \mid \int_{l}^{s} d r_{1} \int_{\mathbb{R}} \frac{\partial G_{\alpha}}{\partial y}\left(r_{1}, z_{1} ; s, y\right) \\
& \left.\cdot \frac{\partial f}{\partial z}\left(r_{1}, z_{1}, u\left(r_{1}, z_{1}\right)\right) D_{l, v} u\left(r_{1}, z_{1}\right) d z_{1}\right|^{2}
\end{aligned}
$$

for $s \in[t-r, t]$ and $y \in \mathbb{R}$. Then, by (5.1) and (3.2), we have

$$
\mathcal{J}_{r}(s, y) \leq 2\left(\mathcal{J}_{r, 1}(s, y)+\mathcal{J}_{r, 2}(s, y)\right)
$$

for $s \in[t-r, t]$ and $y \in \mathbb{R}$. By some elementary calculations one can show that

$$
\begin{aligned}
\mathcal{J}_{r, 1}(s, y) \leq & C \int_{t-r}^{t} d l \int_{\mathbb{R}}\left(|s-l|^{-1} e^{-\frac{C|y-\nu|^{2}}{|s-l|}}+\left(|s-l|^{-\frac{1}{2}} \wedge \frac{|s-l|}{|y-\nu|^{1+\alpha}}\right)^{2}\right. \\
& \left.+2|s-l|^{-\frac{1}{2}} e^{-\frac{C|y-\nu|^{2}}{|s-l|}}\left(|s-l|^{-\frac{1}{2}} \wedge \frac{|s-l|}{|y-\nu|^{1+\alpha}}\right)\right) d \nu \leq C r^{\frac{\alpha-1}{2}}
\end{aligned}
$$

and

$$
\mathcal{J}_{r, 2}(s, y) \leq C \int_{t-r}^{s} \sup _{z_{1} \in \mathbb{R}} \mathcal{J}_{r}\left(r_{1}, z_{1}\right) d r_{1} \leq C r^{\frac{\alpha-1}{2}}+C \int_{t-r}^{s} \sup _{z_{1} \in \mathbb{R}} \mathcal{J}_{r, 2}\left(r_{1}, z_{1}\right) d r_{1}
$$

for $s \in[t-r, t]$ and $y \in \mathbb{R}$. Thus, (5.5) follows from Gronwall's inequality.

Proof of Theorem 5.2 Let $(t, x) \in[0, T] \times \mathbb{R}$. We will adopt a technical argument proposed by Cardon-Weber [29]. By Proposition 2.5, we need only to prove

$$
\|D u(t, x)\|_{\mathcal{H}}>0
$$

almost surely. Recall that the statement $\|D u(t, x)\|_{\mathcal{H}}>0$ is equivalent to the statement $\|D u(t, x)\|_{L^{2}([0, T] \times \mathbb{R})}>0$. Thus, we need only to introduce $\|D u(t, x)\|_{L^{2}([0, T] \times \mathbb{R})}>0$ almost surely. For $0<r<t$ and $x \in \mathbb{R}$, we denote

$$
\Lambda^{1}(t, x, r)=\int_{t-r}^{t} \int_{\mathbb{R}}\left|G_{\alpha}(v, z ; t, x)\right|^{2} d z d v
$$

and

$$
\Lambda^{2}(t, x, r)=\int_{t-r}^{t} d l \int_{\mathbb{R}} d v\left|\int_{l}^{t} d r_{1} \int_{\mathbb{R}} \frac{\partial G_{\alpha}}{\partial z_{1}}\left(r_{1}, z_{1} ; t, x\right) \frac{\partial f}{\partial z_{1}}\left(r_{1}, z_{1}, u\left(r_{1}, z_{1}\right)\right) D_{l, v} u\left(r_{1}, z_{1}\right) d z_{1}\right| .
$$

It follows from (5.1) that

$$
\int_{0}^{t} d l \int_{\mathbb{R}}\left|D_{l, v} u(t, x)\right|^{2} d v \geq C\left(\Lambda^{1}(t, x, r)-\Lambda^{2}(t, x, r)\right)
$$

for all $0<r<t$ and $x \in \mathbb{R}$.

Now, let us to estimate $\Lambda^{1}(t, x, r)$ and $\Lambda^{2}(t, x, r)$. Similar to the proof of (5.6), one can see that

$$
\Lambda^{1}(t, x, r)=C r^{\frac{\alpha-1}{2}}
$$


By (3.8) and Lemma 5.1, one can also see that

$$
\begin{aligned}
E\left|\Lambda^{2}(t, x, r)\right| & \leq \int_{t-r}^{t} d r_{1} \int_{\mathbb{R}} d z_{1} \frac{\partial G_{\alpha}}{\partial z_{1}}\left(t-r_{1} ; x, z_{1}\right) E\left(\int_{t-r}^{r_{1}} \int_{\mathbb{R}}\left|D_{r, z} u\left(r_{1}, z_{1}\right)\right|^{2} d z d r\right) \\
& \leq C r^{\frac{\alpha-1}{2}} \int_{t-r}^{t} d r_{1} \int_{\mathbb{R}} \frac{\partial G_{\alpha}}{\partial z_{1}}\left(t-r_{1} ; x, z_{1}\right) d z_{1} \leq C r^{\alpha-1}
\end{aligned}
$$

for $0<r<t$ and $x \in \mathbb{R}$. Combining this with (5.6), (5.7), and (5.8), we get

$$
\begin{aligned}
& P\left(\int_{0}^{t} \int_{\mathbb{R}}\left|D_{v, z} u(t, x)\right|^{2} d z d v>0\right) \\
& \quad \geq \sup _{r \in\left(0, r_{0}\right]} P\left(C\left(\Lambda^{1}(t, x, r)-\Lambda^{2}(t, x, r)\right)>0\right) \\
& \quad \geq \sup _{r \in\left(0, r_{0}\right]} P\left(\Lambda^{2}(t, x, r) \leq C \Lambda^{1}(t, x, r)\right) \\
& \quad \geq 1-C \inf _{r \in\left(0, r_{0}\right]}\left\{\frac{1}{\left.r^{\frac{\alpha-1}{2}} E\left|\Lambda^{2}(t, x, r)\right|\right\} \geq 1-C \inf _{r \in\left(0, r_{0}\right]} r^{\frac{\alpha-1}{2}}=1}\right.
\end{aligned}
$$

for all $r_{0}>0$, and the theorem follows.

\section{Competing interests}

The authors declare that they have no competing interests.

\section{Authors' contributions}

LTY and DFX carried out the mathematical studies, participated in the sequence alignment, drafted the manuscript, and participated in the design of the study and performed proofs of the results. All authors read and approved the final manuscript.

\section{Author details}

${ }^{1}$ College of Information Science and Technology, Donghua University, Shanghai, 201620, P.R. China. ${ }^{2}$ School of Mathematics and Physics, Anhui Polytechnic University, Wuhu, Anhui 241000, P.R. China. ${ }^{3}$ Department of Mathematics, College of Science, Donghua University, Shanghai, 201620, P.R. China.

\section{Acknowledgements}

The project was sponsored by the National Natural Science Foundation of China $(11571071,71571001)$ and the Natural Science Foundation of Anhui Provincial (1608085MA02).

Received: 5 September 2016 Accepted: 19 December 2016 Published online: 05 January 2017

\section{References}

1. Balan, R, Conus, D: A note on intermittency for the fractional heat equation. Stat. Probab. Lett. 95, 6-14 (2014)

2. Song, J: On a class of stochastic partial differential equations (2015). arXiv:1503.06525v2

3. Chen, $X, H u, Y$, Song, J: Feynman-Kac formula for fractional heat equations driven by fractional white noises (2012). arXiv:1203.0477

4. $\mathrm{Hu}, \mathrm{Y}$, Nualart, D, Song, J: Feynman-Kac formula for heat equation driven by fractional white noises. Ann. Probab. 39, 291-326 (2011)

5. $\mathrm{Hu}, \mathrm{Y}, \mathrm{Lu}, \mathrm{F}, \mathrm{Nualart}, \mathrm{D}$ : Feynman-Kac formula for the heat equation driven by fractional noises with Hurst parameter $H<1 / 2$. Ann. Probab. 40, 1041-1068 (2012)

6. Bo, L, Jiang, Y, Wang, Y: On a class of stochastic Anderson models with fractional noises. Stoch. Anal. Appl. 26, 256-273 (2008)

7. Diop, $M$, Huang, J: Retarded neutral stochastic equations driven by multiplicative fractional Brownian motion. Stoch. Anal. Appl. 32, 820-839 (2014)

8. Duncan, TE, Maslowski, B, Pasik-Duncan, B: Fractional Brownian motion and stochastic equations in Hilbert spaces. Stoch. Dyn. 2, 225-250 (2002)

9. Balan, R: Recent advances related to SPDEs with fractional noise. Prog. Probab. 67, 3-22 (2013)

10. $\mathrm{Hu}, \mathrm{Y}$, Nualart, D: Stochastic heat equation driven by fractional noise and local time. Probab. Theory Relat. Fields 143, 285-328 (2009)

11. Liu, J, Yan, L: On a semilinear stochastic partial differential equation with double-parameter fractional noises. Sci. China Math. 57, 855-872 (2014)

12. Jiang, $Y$, Wang, $X$, Wang, $Y$ : On a stochastic heat equation with first order fractional noises and applications to finance J. Math. Anal. Appl. 396, 656-669 (2012) 
13. Jiang, $Y$, Wei, $T$, Zhou, $X$ : Stochastic generalized Burgers equations driven by fractional noises. J. Differ. Equ. 252, 1934-1961 (2012)

14. Balan, R, Tudor, CA: The stochastic heat equation with a fractional-colored noise: existence of the solution. ALEA Lat. Am. J. Probab. Math. Stat. 4, 57-87 (2008)

15. Balan, RM, Tudor, CA: Stochastic heat equation with multiplicative fractional-colored noise. J. Theor. Probab. 23, 834-870 (2010)

16. Tudor, CA: Recent developments on stochastic heat equation with additive fractional-colored noise. Fract. Calc. Appl. Anal. 17, 224-246 (2014)

17. Sobczyk, K: Stochastic Differential Equations with Applications to Physics and Engineering. Kluwer Academic, London (1991)

18. Droniou, J, Imbert, C: Fractal first-order partial differential equations. Arch. Ration. Mech. Anal. 182, 299-331 (2006)

19. Chen, Z, Kumagai, T: Heat kernel estimates for jump processes of mixed types on metric measure spaces. Probab. Theory Relat. Fields 140, 277-317 (2008)

20. Chen, Z, Kumagai, T: Heat kernel estimates for stable-like processes on $d$-sets. Stoch. Process. Appl. 108, 27-62 (2003)

21. Chen, Z, Kim, P, Song, R: Global heat kernel estimate for relativistic stable processes in exterior open sets. J. Funct. Anal. 263, 448-475 (2012)

22. Chen, Z, Kim, P, Song, R: Heat kernel estimates for $\Delta^{\alpha / 2}+\Delta^{\beta / 2}$. III. J. Math. 54, 1357-1392 (2000)

23. Chen, Z, Kim, P, Song, R: Heat kernel estimates for $\Delta+\Delta^{\alpha / 2}$ in $C^{1,1}$ open sets. J. Lond. Math. Soc. 84, 58-80 (2011)

24. Nualart, D: Malliavin Calculus and Related Topics. Springer, Berlin (2006)

25. Wei, T: High-order heat equations driven by multi-parameter fractional noises. Acta Math. Sin. Engl. Ser. 26, 1943-1960 (2010)

26. Walsh, JB: An introduction to stochastic partial differential equations. In: Ecole d'été de Probabilités de Saint Flour XIV Lecture Notes in Math., vol. 1180, pp. 266-439. Springer, Berlin (1986)

27. Dalang, R: Extending the martingale measure stochastic integral with applications to spatially homogeneous SPDE's. Electron. J. Probab. 4, 1-29 (1999)

28. Zhang, T, Zheng, W: SPDEs driven by space-time white noises in high dimensions: absolute continuity of the law and convergence of solutions. Stoch. Stoch. Rep. 75, 103-128 (2003)

29. Cardon-Weber, C: Cahn-Hilliard stochastic equation: existence of the solution and its density. Bernoulli 7, 777-816 (2000)

\section{Submit your manuscript to a SpringerOpen ${ }^{\circ}$ journal and benefit from:}

- Convenient online submission

Rigorous peer review

- Immediate publication on acceptance

- Open access: articles freely available online

- High visibility within the field

- Retaining the copyright to your article 